\title{
Time-to-passage judgments on circular trajectories are based on relative optical acceleration
}

\author{
DIRK KERZEL \\ Max Planck Institute for Psychological Research, Munich, Germany \\ HEIKO HECHT \\ Massachusetts Institute of Technology, Cambridge, Massachusetts
}

and

NAM-GYOON KIM

University of Connecticut, Storrs, Connecticut

\begin{abstract}
Current theories of arrival time have difficulty explaining performance in the common but neglected case of nonlinear approach. Global tau, a variable supposed to guide time-to-passage (TTP) judgments of objects approaching on linear trajectories, does not apply to circular movement. However, TTP judgments are surprisingly accurate in such cases. We simulated movement through a three-dimensional cloud of point-lights on various circular trajectories. Arrival-time judgments were found to be above chance when observers had to determine which of two expansionless targets would pass them first. Similar to the inside bias observed in heading studies on circular trajectories, observers showed a strong bias to select the target on the inside of their own curved motion path as passing by first. Analysis of the projected target motion revealed that targets on the inside had lower optical velocities and relatively high optical acceleration rates. Empirical TTP judgments agreed best with a strategy based on relative optical velocity changes.
\end{abstract}

Information about the time before an approaching object reaches or comes closest to an observer is essential in many skilled activities, such as in ball games or when walking through a cluttered environment. With few exceptions (e.g., Bootsma \& Oudejans, 1993; Heuer, 1993; Kaiser \& Hecht, 1995; Kaiser \& Mowafy, 1993), linear head-on approaches of objects moving at constant velocity have been investigated (for overviews, see Tresilian, 1991, 1995; Wann, 1996). In the present study, we examine common but neglected bypass situations involving nonlinear motion trajectories, in order to disentangle optical velocity and acceleration, two optical variables that are typically confounded in linear approach scenarios. We simulated movement on circular trajectories through a three-dimensional (3-D) cloud of point-lights. In a relative judgment task, observers had to determine which one of two expansionless targets would pass by first. Thus, global flow information was isolated, since within-object dilation was absent. We demonstrate that in addition to optical velocity, which is used in linear ap-

This work was supported by fellowships from the Studienstiftung des Deutschen Volkes and the Fulbright Commission to the first author. We thank G. J. Andersen, J. Tresilian, A. Wohlschläger, S. F. W. Neggers, and two anonymous reviewers for helpful comments on an earlier version of this manuscript. Correspondence concerning this article should be addressed to D. Kerzel, Max Planck Institute for Psychological Research, Amalienstr. 33, 80799 Munich, Germany (e-mail: kerzel@mpipfmuenchen.mpg.de). proach, relative velocity change acts as a cue to depth in the case of circular approach paths.

A prominent account of performance in situations that require the interception of approaching objects is the tau hypothesis. Lee (1976; Hoyle, 1957) demonstrated that the time to contact (TTC) with an object approaching on a straight path is given by a single optical variable, tau. Tau refers to the instantaneous rate of change in the visual extent of the object, relative to the extent of the instantaneous image. Although empirical evidence in support of observers' ability to use tau has been obtained (e.g., Bootsma \& van Wieringen, 1990; Lee, 1976; Schiff \& Detwiler, 1979; Schiff \& Oldak, 1990; Simpson, 1988; Todd, 1981), some studies have shown that a tau-based mechanism is imperfect at best (Tresilian, 1995). In particular, effects of size (DeLucia, 1991; DeLucia \& Warren, 1994) and relative distance (Law et al., 1993) on time-to-arrival judgments have been demonstrated. The latter findings suggest that observers do not rely on a single optical variable that gives veridical arrival-time estimates but, rather, use multiple cues of a limited environmental validity to estimate the object's distance from the observer. So far, most studies have investigated arrivaltime judgments in situations that involved extended approaching objects, so that within-object tau information was present. In Tresilian's (1991) nomenclature, this is referred to as local tau.

However, in many contexts, local tau information is unavailable or leads to erroneous arrival-time predic- 
tions. For instance, during off-axis approaches, withinobject tau is compromised unless the approaching object is spherical (Kaiser \& Mowafy, 1993). In these situations, observers have been hypothesized to use an optical variable mathematically similar to local tau but operating on the angle between a single object point and the direction of egomotion. Global tau is defined as the angle between object and direction of motion divided by the rate of change of this angle. Tresilian (1991) called this variable global tau because it is instantiated in the global flow field. To isolate observers' ability to judge time to passage (TTP), Kaiser and Mowafy constructed displays that consisted entirely of single-pixel dots. Although object dilation is zero, which specifies a stationary object in natural scenarios, the motion of the dots is readily perceived as approach (e.g., Kaiser \& Mowafy, 1993; Warren, Morris, \& Kalish, 1988). Since cues from expansion of the image were eliminated, local tau was not available as an estimate of the time until closest approach. Kaiser and Mowafy claimed that observers are sensitive to global tau, because relative and absolute TTP judgments were robust. As compared with sensitivity to approaching objects showing object dilation, however, performance was rather poor. Relative TTP judgments were reliable, with differences in TTP of half a second or more. In contrast, Todd (1981) reported 90\% accuracy for differences in TTC of expanding objects for temporal differences as small as $150 \mathrm{msec}$. Kaiser and Mowafy suggested that the greater sensitivity to TTC, as compared with TTP, results from the different functional roles of the two types of information. Whereas TTC is used for precise movements, such as catching, TTP is important for global navigation. Moreover, the identification of the direction of heading requires an analysis of the global flow field that may be more demanding than the extraction of local image changes.

Furthermore, it has been demonstrated that global flow information may overrule local object dilation. DeLucia and Novak (1997) examined the effects of set size in a relative judgment task in which observers had to indicate which of 2-8 objects approaching the observer would be first to pass. The objects showed both local and global expansion. A larger drop in observers' performance was noted when global expansion rate contradicted time-to-arrival information, as compared with cases in which local expansion rate contradicted time-toarrival information. Therefore, a further examination of the type of global information that enters observers' TTP judgments appeared to be justified.

In line with studies demonstrating that single optical cues are used to estimate arrival time, Kerzel, Hecht, and Kim (1999) found that reliable TTP judgments may be achieved without sensitivity to global tau. Instead, observers' judgments were guided by a related but simpler variable, the optical velocity of the object. Consider an object offset by a constant distance, $x$, from the observer's linear trajectory. The optical angle, $A$, between the observer's heading and the object may be calculated, using the law of small angles, as

$$
A=\frac{x}{t * v},
$$

where $t$ denotes time and $v$ the constant velocity of the approaching object. Differentiation with respect to time yields the optical velocity, $A^{\prime}$. As can be seen in the formula, $A^{\prime}$ depends on the offset $x$ :

$$
A^{\prime}=-\frac{x}{t^{2} * v} \text {. }
$$

In contrast, global tau (GT) is independent of target offset:

$$
G T=\frac{A}{A^{\prime}} .
$$

Thus, global tau and optical velocity may be brought into conflict by varying the lateral offsets of the targets. Whereas optical velocity increases with offset, global tau does not. Figure 1 shows the optical velocities of targets, approaching the observer on a linear trajectory, that are offset by a certain distance from the direction of motion. As is evident in the figure, optical velocity increases with lateral offset of the targets. When asked to judge which one of the two targets will pass by first, observers showed a strong tendency to select the target with the larger offset and, therefore, the largest optical velocity. This tendency is at odds with the supposed ability to use global tau, because global tau values are independent of target offset. Rather, the subjects may have used optical velocity as a cue to depth.

However, further analysis of the motion parameters involved in linear approach shows that optical velocity is confounded with another variable, the optical acceleration of the target. Similar to optical velocity, optical acceleration $A^{\prime \prime}$ depends on lateral target offset:

$$
A^{\prime \prime}=\frac{2 * x}{t^{3} * v}
$$

Therefore, one may call into question the claim that observers used optical velocity as a cue to depth. Figure 1 shows that the acceleration of targets at different distances from the direction of motion is proportional to their optical velocity - that is, the ratio of $A^{\prime \prime}$ and $A^{\prime}$ does not vary with lateral offset:

$$
\frac{A^{\prime \prime}}{A^{\prime}}=-\frac{2}{t} \text {. }
$$

Thus, relative optical acceleration may be another cue to depth that indicates TTP with a higher validity than optical velocity, because it is not affected by target offset.

In the present study, we examined whether observers are able to make reliable judgments of TTP on circular trajectories and what informational quantities they use in doing so. To this end, we used approaches on nonlinear 3-D trajectories where optical velocity and acceleration do not increase proportionally as a function of offset. Using the same time-to-passage paradigm as Kaiser 


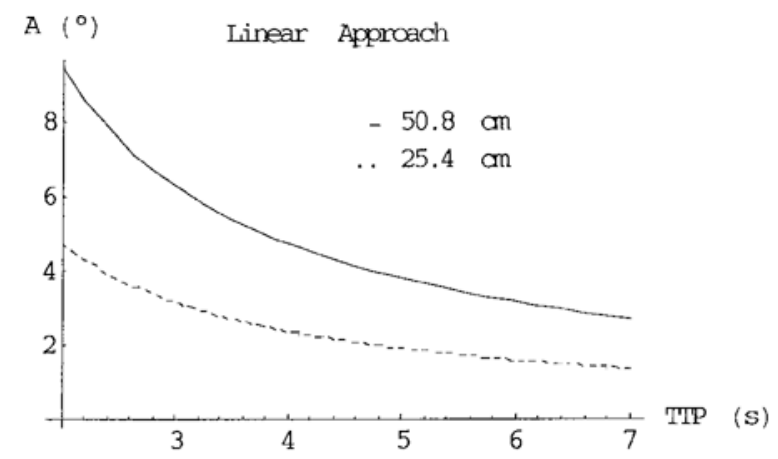

$A^{\prime}(\% / S)$

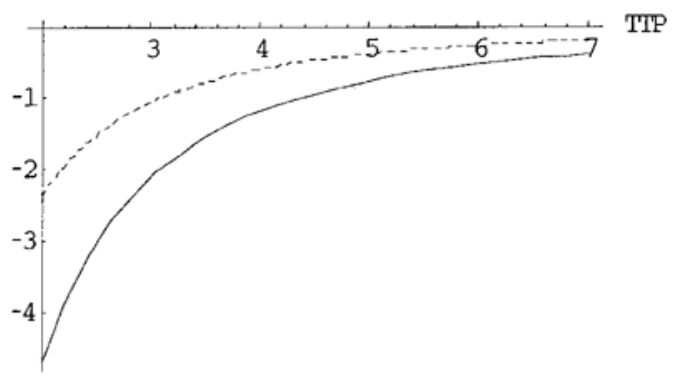

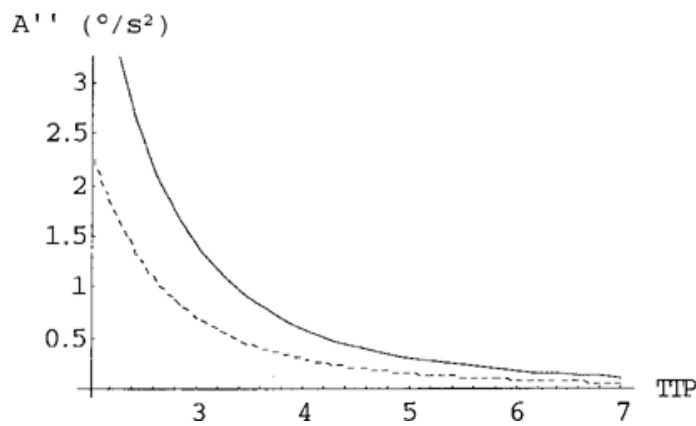

(s)

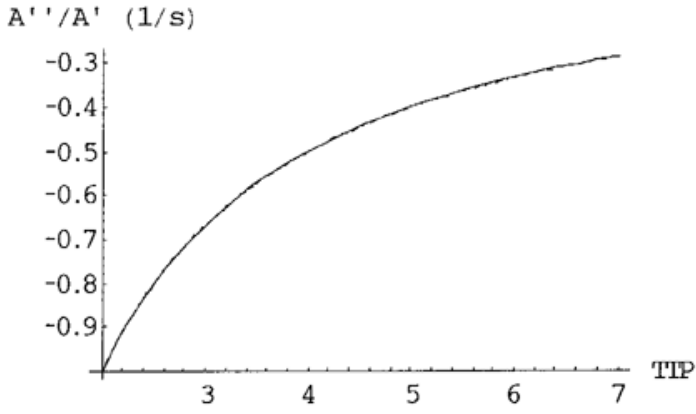

Figure 1. The optical angle $A$ between object and direction of motion as a function of time to passage (TTP). Objects approaching the observer until 2 sec before passage are simulated. The objects were offset by $25.4 \mathrm{~cm}$ (dotted line) and $50.8 \mathrm{~cm} . A^{\prime}$ and $A^{\prime \prime}$ are the derivatives with respect to time-that is, optical velocity and optical acceleration of $A$, respectively. $A^{\prime \prime} / A^{\prime}$ is referred to as relative optical acceleration.

and Mowafy (1993), we simulated observer motion on a circular trajectory through a 3-D cloud (see Figure 2). To our knowledge, TTP judgments on circular trajectories that involve a rotation around the vertical axis have not been studied before. So far, studies simulating observer motion on circular trajectories have only examined observers' abilities to perceive their direction of motion (heading; Cutting, 1986; Kerzel \& Hecht, 1997; Kim \& Turvey, 1998; Stone \& Perrone, 1997; Warren, Mestre, Blackwell, \& Morris 1991). These studies showed that observers were able to judge their heading on circular trajectories reliably, albeit with less accuracy than on linear trajectories and with a bias to perceive heading in a 3-D cloud toward the inside of the trajectory (but see Kim \& Turvey, 1998, for a failure to obtain this effect). Some sparse evidence is available on TTC judgments during rotation around the body axis (Lee, Young, \& Rewt, 1992) and for rotating objects (DeLucia \& Liddell, 1998, note 9).

In our analysis of TTP on circular trajectories, we focus on the temporal characteristics of the optical angle, $A$, subtended by an object approaching the observer and the tangential direction of motion. Projectively, a moving target approaching a stationary observer is equivalent to a moving observer approaching a stationary target. We will use these two formulations interchangeably. With the law of small angles, $A$ may be calculated for a given radius, $r$, of the target trajectory, for a constant distance, $x$, of the observer with respect to the center of rotation, and for the constant angular velocity, $w$, of the target (see Figure 2A):

$$
A=\frac{x-r * \cos (w * t)}{r * \sin (w * t)} .
$$

Differentiation yields the optical velocity, $A^{\prime}$, and optical acceleration, $A^{\prime \prime}$, as shown in Equations 7 and 8 at the bottom of this page.

In our displays, we simulated the motion of an inside target traveling on radius $r 1$ ( $r 1=x-$ offset $)$ and an outside target on radius $r 2(r 2=x+$ offset $)$. Owing to the highly nonlinear nature of circular approaches, the relationship between inside and outside targets depends on

$$
\begin{gathered}
A^{\prime}=\frac{w * r-x * w * \cos (w * t)}{r * \sin ^{2}(w * t)} \\
A^{\prime \prime}=-\frac{w * \csc (t * w) *\left[-w * x+2 * w * x * \cot ^{2}(t * w)+\csc ^{3}(t * w)\right]}{r^{3}} .
\end{gathered}
$$



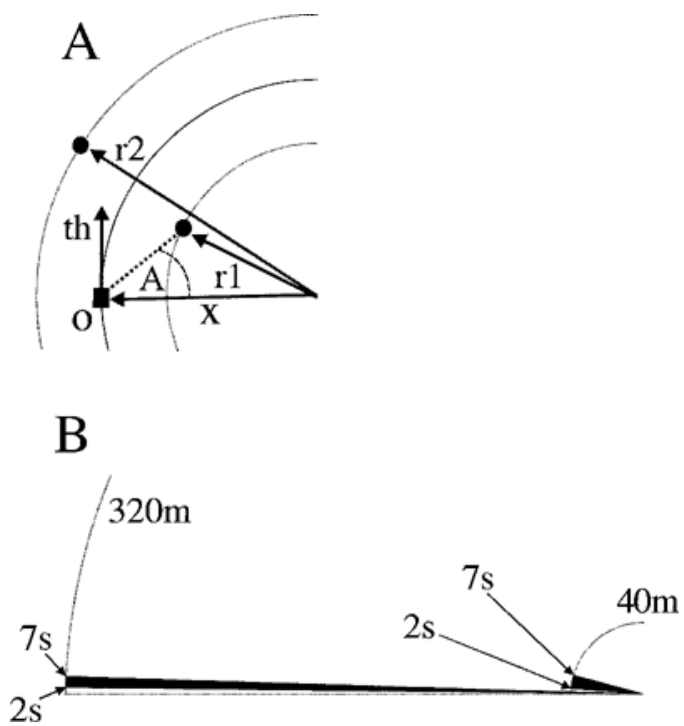

Figure 2. Schematic drawing of the simulated stimulus space (top view). Panel A: An observer, $o$, is at a distance $x$ from the center of rotation. The observer's instantaneous heading direction, $t h$, is tangential to his circular path. Two targets approach the observer on radii $r 1$ and $r 2$. Since $r 1<x$ and $r 2>x$, the target on $r 1$ is referred to as the inside target, and the target on $r 2$ as the outside target. The optical angle $A$ is subtended by an object and the instantaneous tangential heading direction. In our displays, tangential heading direction was coincident with direction of gaze. Panel B: The angular distance traversed during the display duration from 7 to 2 sec before passage. Since the tangential velocity was constant, the targets traversed a larger angular distance on the small circular trajectory (about $11^{\circ}$ ) than on the large trajectory (about $1.4^{\circ}$ ). Angular distances and size of the circles are drawn to scale.

which part of the trajectory is considered. Here, we focus on the most relevant scenario in which the targets are approximately straight ahead of the observer-that is, within a limited field of view (see Figure 2). In particular, situations in which the outside target traverses the observer's straight ahead view $[x=r 2 * \cos (w * t), A=0]$ and the velocity of the inside target reaches zero $[w * r 1=$ $\left.x * w * \cos (w * t), A^{\prime}=0\right]$ offer some interesting characteristics. The nonlinearities involved in this scenario may be observed when the target traverses a substantial angular distance around these critical points. In our displays, we kept the tangential velocity, $v$, constant, so that the angular velocity, $w$, varied as a function of radius given that $w=v / r$. Thus, targets on small trajectories traversed a larger angular distance, so that the optical projection showed highly nonlinear behavior around the critical points. With large trajectories, the angular velocity was small, so that the targets were placed beyond these points to have the same final TTP as targets on small trajectories (see Figure 2B). Therefore, targets on large trajectories behaved in a manner very similar to that for linear movement for the angular distances considered. For targets traveling on small trajectories around the critical points, the following observations can be made (see Figure 3). (1) The optical angle $A$ is larger for inside targets than for outside targets. (2) The velocity profiles are very similar for inside and outside targets; however, absolute velocities are lower for inside targets. (3) Acceleration profiles and absolute acceleration values are almost identical for inside and outside targets. (4) In contrast to linear approach, relative acceleration $\left(A^{\prime \prime} / A^{\prime}\right)$ does not eliminate dependency on target offset (i.e., $x-r$ ). Relative acceleration profiles and absolute values are highly dissimilar for inside and outside targets. Whereas there is a steady increase of absolute relative acceleration for outside targets, values are more variable for inside targets. Furthermore, absolute relative acceleration is higher for inside targets. (5) Global tau does not give the correct temporal order for inside and outside targets. Since the optical velocity of inside targets is lower than that of outside targets, absolute global tau values will be higher for inside targets, irrespective of temporal separation. The failure of global tau to specify TTP on circular approaches is not surprising, since linear approach has been assumed in all of its derivations (see, e.g., Tresilian, 1991).

These properties give rise to a number of predictions. (1) If observers exclusively judge TTP on the basis of optical velocity, as was suggested by Kerzel et al. (1999), outside targets should be judged to arrive sooner than inside targets. (2) If observers rely on optical acceleration, no systematic difference between inside and outside targets would be predicted. (3) If observers rely on relative acceleration, inside targets should be perceived to be closer. (4) If global tau guides TTP judgments, outside targets should be judged as arriving first. (5) If observers judge the most eccentric target to pass by first, they should select the inside target more often than the outside target. Previous research has ruled out the last hypothesis. Kerzel et al. (1999, Experiments 3-4) varied the angle between direction of motion and direction of gaze, which displaced the targets to the left or right of the screen. Observers showed no tendency to select the target further to the left or right; rather, relative TTP judgments were based on optical velocity, irrespective of eccentricity. Also, when targets were placed on the same side with respect to the direction of motion (Kaiser \& Mowafy, 1993), observers did not select the outer target, but the target with the higher optical velocity (see Kerzel et al., 1999, for a discussion of the relative motion conditions in Kaiser and Mowafy, 1993).

\section{EXPERIMENT 1 \\ Relative Passage Judgments on Small Circular Trajectories}

To test predictions derived from usage of different optical variables, movement on circular trajectories with radii of 40,80 , and $160 \mathrm{~m}$ was simulated. Targets were displaced to the inside and outside of the virtual trajectory by a certain distance. Radial distance between the objects was manipulated to yield differences in TTP of 
A ( $\left.{ }^{\circ}\right)$

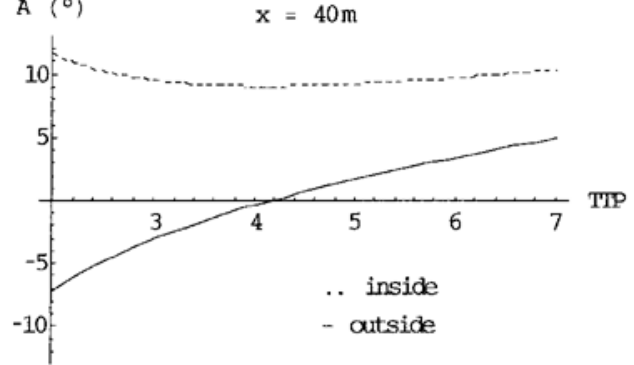

$\mathrm{A}^{\prime}(\% / \mathrm{s})$

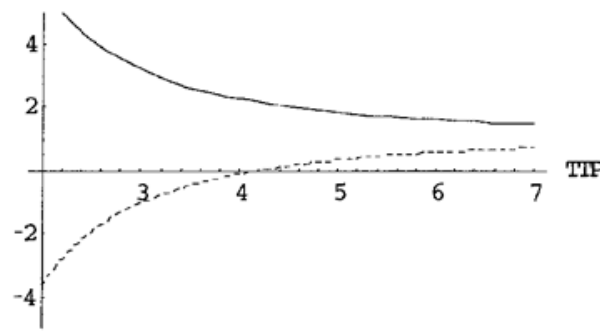

$A^{\prime \prime}\left(\% / S^{2}\right)$

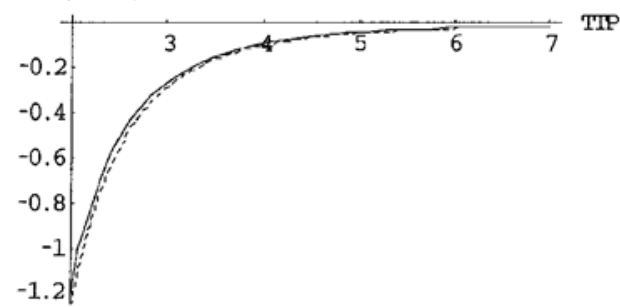

$A^{\prime} ' / A^{\prime}(1 / s)$

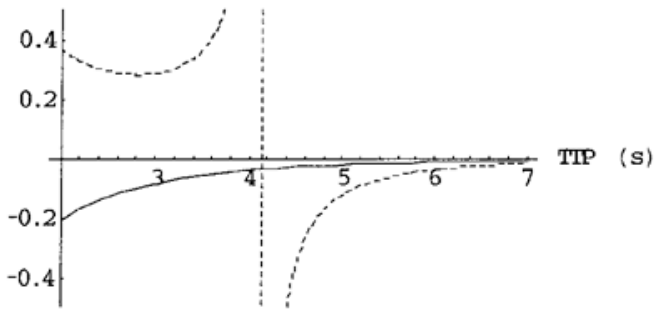

Global Tau (s)

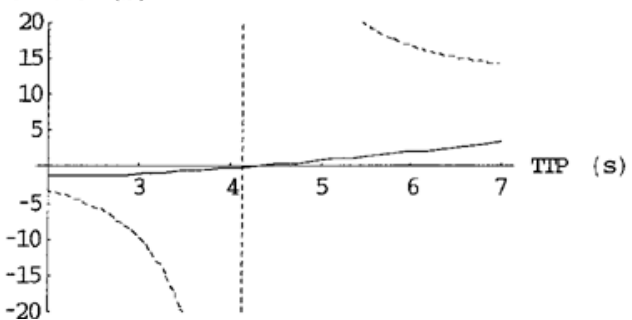

(s)

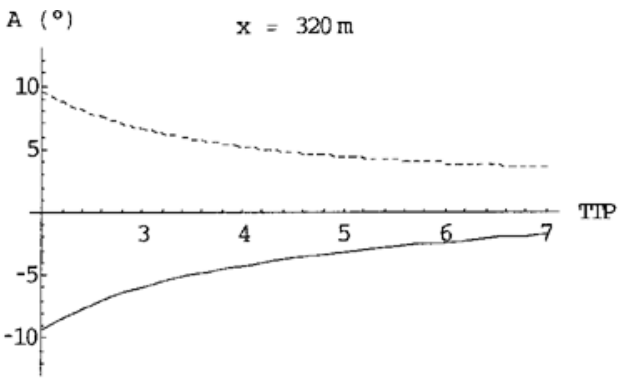

(s) (s)

(S)

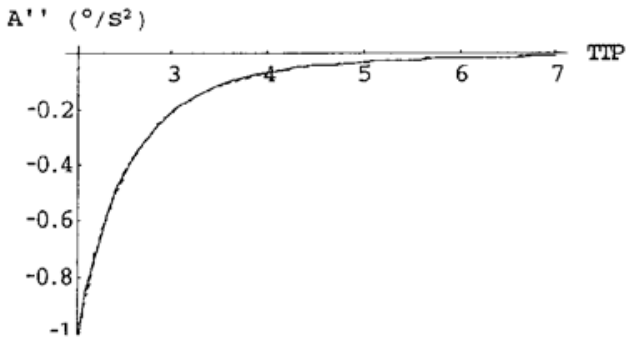

(s)
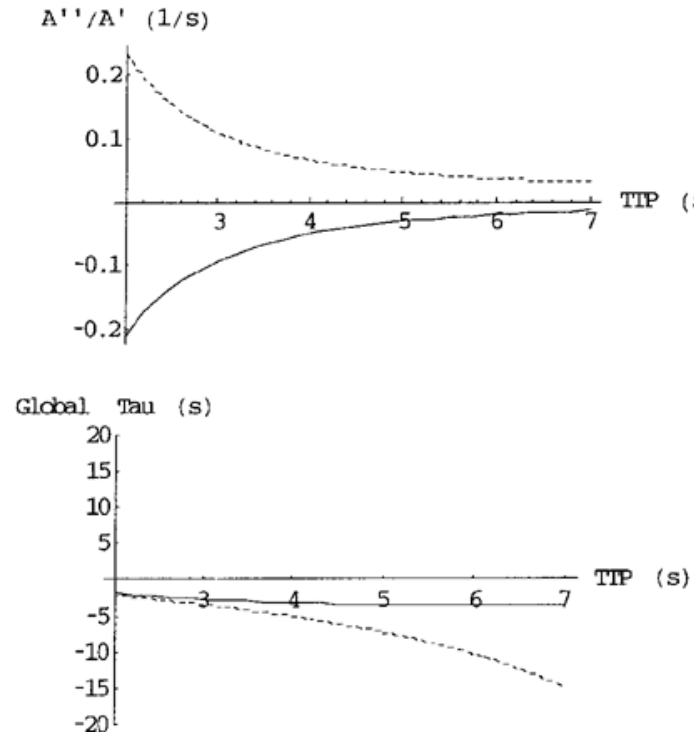

Figure 3. The angle $A$ between object and tangential direction of motion is graphed for two objects approaching an observer on a small $(x=40 \mathrm{~m})$ or a large $(x=320 \mathrm{~m})$ trajectory. The inside target (dotted line) has a radius smaller than the distance of the observer to the center of rotation $(r 1=x-0.508 \mathrm{~m})$; the outside target (solid line) has a larger $\operatorname{radius}(r 2=x+0.508 \mathrm{~m})$. If one thinks of positive values of $A$ as subtending toward the right of the observer's straight ahead view, then a rightward turn is simulated (as in Figure 2). The outside target crosses the line of sight of the observer from right to left, whereas the inside target stays to the right of the observer. The temporal derivatives of $A$ are graphed. $A^{\prime}$ denotes the optical velocity of $A$, $A^{\prime \prime}$ the optical acceleration, and $A^{\prime \prime} \mid A^{\prime}$ the relative optical acceleration. Global tau is the ratio of $A$ and $A^{\prime}$. Note that global tau values do not specify time to passage (TTP) accurately. Also, inside targets have relatively low optical velocity, as compared with their acceleration rates. The difference between inside and outside targets diminishes with a radius of $320 \mathrm{~m}$, since we kept the tangential velocity constant, so that the angular velocity was lower on large trajectories. 
$0.25,0.5,0.75$, and $1 \mathrm{sec}$. As was outlined above, movement on circular trajectories provides the opportunity to disentangle two optical variables that are confounded in linear approaches. Thus, we may learn which cues observers use to estimate TTP. An account in terms of multiple optical cues deviates from theories claiming that observers use single, higher order variables specifying arrival time (e.g., Gibson, 1966). In particular, we do not claim that sensitivity to TTP of targets on circular trajectories reveals extraction of higher order variables specifying TTP. Rather, above-chance performance may result from the use of multiple cues of limited environmental validity. Reliance on such cues may be revealed in cases in which these cues fail and produce a bias.

\section{Method}

Subjects. Nine undergraduate students at the University of Connecticut participated in partial fulf illment of a course requirement. All the subjects had normal or corrected-to-normal vision.

Stimuli. The stimuli were presented on a $48-\mathrm{cm}$ (diagonal) screen drawn at a refresh rate of $60 \mathrm{~Hz}$ by a Silicon Graphics Personal Indigo 2 workstation. The display had a resolution of $1,280 \times$ 1,024 pixels (horizontal by vertical) and subtended $47^{\circ} \times 36^{\circ}$ (horizontal by vertical). Stimulus displays simulated observer rotation through a 3-D cloud of 600 white single-pixel dots. Arrangement of the dots was accomplished in the following way. First, a rectangular cloud was created as in Kaiser and Mowafy (1993). It was $17.37 \mathrm{~m}$ deep, starting from the clipping plane $(z=4.86 \mathrm{~m})$, and extended $5 \mathrm{~m}$ horizontally and vertically from the observer. Dots were placed on a grid, and some random jitter was added. Then, the polar coordinates of the dots were determined. The angle between observer and dot with respect to the center of rotation was used as azimuth, and the $x$-coordinate with respect to the center of rotation as radius. Therefore, the dots were placed around the observer's cir- cular path. Because azimuth values were derived from a rectangular stimulus space, the maximal angular distance between dot and observer was slightly larger for dots on the inside than for dots on the outside. The maximal value of this difference was $6^{\circ}$ of rotation with the smallest radius. Each display lasted $5 \mathrm{sec}$ (300 frames). Dots that went out of sight were not replaced. The observer's tangential velocity was $2.54 \mathrm{~cm}$ per frame, or $1.52 \mathrm{~m} / \mathrm{sec}$. Direction of gaze was tangential to the circular trajectory and coincided with the instantaneous tangential direction of motion (heading). Both vectors passed through the center of the screen. The angular distance between observer and two colored targets was determined so that the closer of the two would pass $2 \mathrm{sec}$ after display termination. The targets were offset from the observer's trajectory toward the inside and outside of the virtual circle (see Figure 2).

Design. A four-factor within-subjects design was used. The first factor was the radius of the observer's trajectory. It was varied randomly between 40,80, and $160 \mathrm{~m}$. The second factor was TTP difference. The angular separation with respect to the center of rotation between the two targets was determined so as to give rise to differences in TTP of $0.25,0.5,0.75$, and $1 \mathrm{sec}$, with the close target $2 \mathrm{sec}$ to passage at the point of display termination. The third factor was the relative position of the leading target with regard to the observer's trajectory. The target that would pass the observer first was placed equally often on a trajectory with a radius smaller than the observer's (i.e., on the inside), as on a trajectory with a larger radius (i.e., on the outside). The fourth factor was the distance of the leading target from the observer's trajectory. The two targets were displaced by either 25.4 or $50.8 \mathrm{~cm}$ to opposite sides of the observer's trajectory, which yielded four possible target placements. The four TTP differences, three radii, two positions of the leading target, and four target placements were fully crossed (96 cases) and duplicated by reversal of the direction of rotation (192 cases).

Procedure. The subjects were seated in a dimly lit room about $44 \mathrm{~cm}$ from the CRT screen and watched the displays binocularly. A chinrest was used. The subjects were told that they would be watching stimuli simulating their own movement through a 3-D

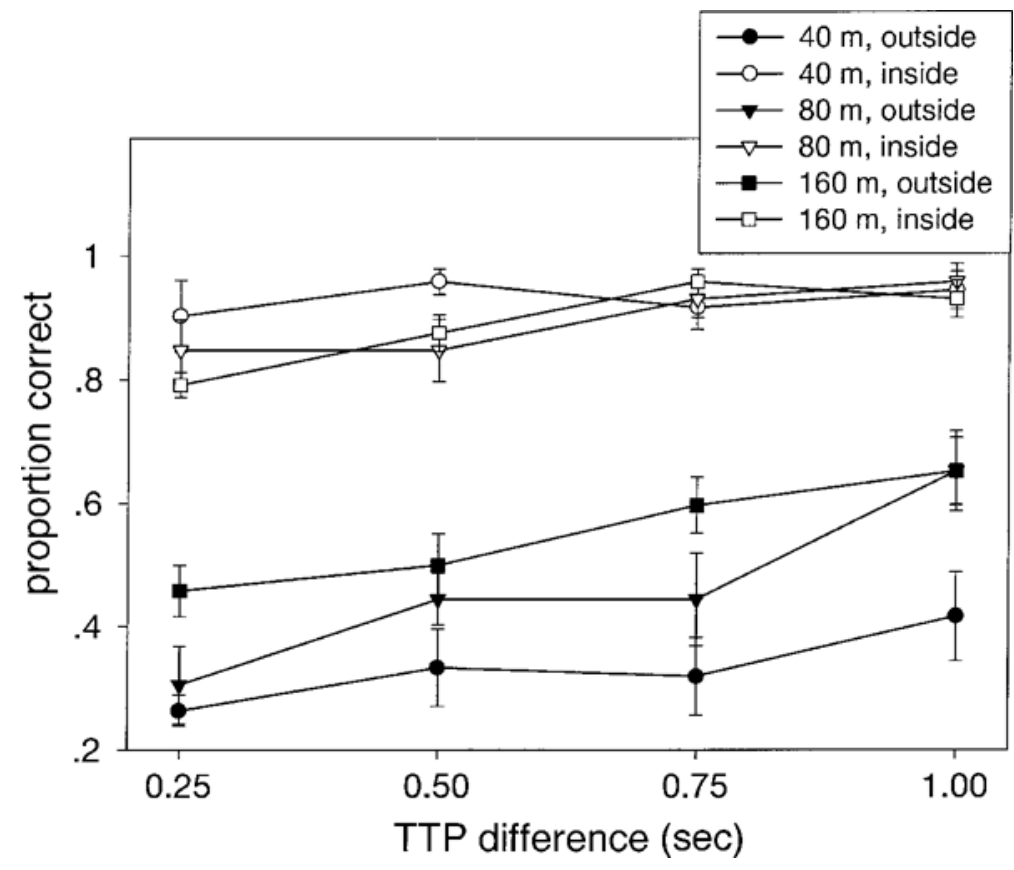

Figure 4. Mean proportion of correct responses and standard error (between subjects) as a function of relative position of the leading target, radius, and difference in time to passage (TTP) in Experiment 1. 
Table 1

Significant Within-Subjects Effects in Experiments 1, 2, 3, and 4

\begin{tabular}{|c|c|c|c|}
\hline Effect & $F$ & $d f$ & $p$ \\
\hline \multicolumn{4}{|l|}{ TTP } \\
\hline Experiment 1 & 10.47 & 3,24 & .0001 \\
\hline Experiment 2 & 22.66 & 3,24 & .0001 \\
\hline Experiment 3 (DS) & 4.21 & 3,24 & .0158 \\
\hline Experiment 3 (SS) & 10.15 & 3,24 & .0002 \\
\hline Experiment 4 & 7.98 & 3,21 & .001 \\
\hline \multicolumn{4}{|l|}{ Radius } \\
\hline Experiment 1 & 18.08 & 2,16 & .0001 \\
\hline Experiment 2 & 8.35 & 2,16 & .0033 \\
\hline Experiment 4 & 8.56 & 2,14 & .0037 \\
\hline \multicolumn{4}{|l|}{ Relative position } \\
\hline Experiment 1 & 274.03 & 1,8 & .0001 \\
\hline Experiment 2 & 86.43 & 1,8 & .0001 \\
\hline Experiment 3 (DS) & 38.42 & 1,8 & .0003 \\
\hline Experiment 4 & 209.74 & 1,7 & .0001 \\
\hline \multicolumn{4}{|l|}{ Distance } \\
\hline Experiment 1 & 74.22 & 1,8 & .0001 \\
\hline Experiment 2 & 131.11 & 1,8 & .0001 \\
\hline Experiment 3 (DS) & 33.80 & 1,8 & .0004 \\
\hline Experiment 3 (SS) & 7.97 & 1,8 & .0224 \\
\hline Experiment 4 & 41.58 & 1,7 & .0004 \\
\hline \multicolumn{4}{|c|}{ Distance $\times$ relative position } \\
\hline Experiment 2 & 22.06 & 1,8 & .0002 \\
\hline Experiment 3 (DS) & 32.11 & 1,8 & .0001 \\
\hline Experiment 4 & 66.61 & 1,7 & .0001 \\
\hline \multicolumn{4}{|c|}{ Radius $\times$ relative position } \\
\hline Experiment 1 & 18.05 & 2,16 & .0001 \\
\hline Experiment 2 & 106.43 & 2,16 & .0001 \\
\hline Experiment 3 (DS) & 6.71 & 2,16 & .0077 \\
\hline Experiment 4 & 23.06 & 2,14 & .0001 \\
\hline \multicolumn{4}{|l|}{$\mathrm{TTP} \times$ relative position } \\
\hline Experiment 2 & 3.62 & 3,24 & .0275 \\
\hline Experiment 4 & 6.71 & 3,21 & .0024 \\
\hline \multicolumn{4}{|l|}{ TTP $\times$ distance } \\
\hline Experiment 1 & 3.20 & 3,24 & .0413 \\
\hline Experiment 2 & 7.00 & 3,24 & .0015 \\
\hline Experiment 3 (SS) & 8.37 & 3,24 & .0005 \\
\hline \multicolumn{4}{|l|}{ TTP $\times$ radius } \\
\hline Experiment 4 & 2.61 & 6,42 & .0304 \\
\hline \multicolumn{4}{|l|}{ Radius $\times$ distance } \\
\hline Experiment 1 & 4.96 & 2,16 & .0211 \\
\hline Experiment 4 & 4.02 & 2,14 & .0418 \\
\hline
\end{tabular}

Note-Proportion of correct responses was the dependent measure. Separate analyses of variance were run in Experiment 3 for different side (DS) and same side (SS) target placements. TTP, time-to-passage.

cloud of white dots. Their task was to judge which one of the two colored target dots would pass them first if the movement were to continue. The subjects initiated a trial by means of a keypress. On each trial, the first frame of the display was shown for $2 \mathrm{sec}$ to ensure that the subjects were able to locate the targets. The displays remained visible until the subjects pressed one key if they thought that the left target would pass by them first and another key if they thought that the right target would pass by first. Feedback was provided after each trial, in both the practice and the experimental trials. To familiarize the subjects with the task, 16 practice trials randomly drawn from the 192 experimental trials were presented.

\section{Results}

Proportions correct. Mean proportion of correct responses (PCR) are graphed in Figure 4. A four-way within-subjects analysis of variance (ANOVA; TTP $\times$ radius $\times$ relative position of leading target $X$ distance of leading target) was conducted. The results are presented in Table 1. It was confirmed that performance increased with TTP difference from .59 to .76 . By $t$ test, proportions correct were well above chance for all TTP differences $(p s<.0001)$. PCR increased with the size of the radius from .63 at the smallest to .72 at the largest. Judgments were more accurate when the leading target was on the inside than when it was on the outside of the observer's trajectory (.91 vs. .45). Accuracy was lower when the leading target was close to the observer's trajectory (.64 vs. .74). The interaction of radius and the relative position of the leading target was significant, indicating that the advantage of trials with the leading targets on the inside decreased from .6 at $40 \mathrm{~m}$ to .34 at $160 \mathrm{~m}$. The difference in accuracy between close and far target placement decreased with TTP from .21 at $0.25 \mathrm{sec}$ temporal separation to .07 at $1 \mathrm{sec}$ and increased with radius from .07 at $40 \mathrm{~m}$ to .18 at $160 \mathrm{~m}$. Overall, there was a strong bias to judge the inside target as passing by first $(73 \%$ of all trials, different from $50 \%, p<.0001)$. Furthermore, the target far from the observer's trajectory was judged to pass by first more often than the close target $(56 \%$ of all trials, different from $50 \%, p<.0001$ ).

$\boldsymbol{d}^{\prime}$ and bias. A signal detection analysis was carried out with respect to the relative position of the target. Judgments that the inside target passed by first when this was the case were considered hits, and judgments that the inside target passed by first when it did not were considered false alarms. The hit rate $[\mathrm{H}=\mathrm{P}$ ("inside first" $<$ inside first, outside second $>$ )] and the false alarm rate [FA = P ("inside first" $\mid<$ outside first, inside second $>$ ) ] were computed. The $d^{\prime}$ values were adjusted for usage of a two-alternative forced-choice procedure by multiplication with 1/SQRT(2) (Macmillan \& Creelman, 1991). Because of the strong bias effects with small radii-that is, a high percentage of "inside first" judgments-hit rates of one occurred occasionally. Since a $z$ value is not defined for a probability of one, conditions with hit rates of one were treated as missing values. False alarm rates never reached zero. Using standard detection theory (Macmillan \& Creelman, 1991), bias was calculated as $-0.5 *[z(\mathrm{H})+z(\mathrm{~F})]$. Across all conditions, mean $d^{\prime}$ was $0.87(S E=0.11)$ and mean bias was $-0.53(S E=0.04)$. The $d^{\prime}$ and bias values were calculated for each radius and for each TTP difference, and one-way ANOVAs were run on $d^{\prime}$ (Table 2) and bias (Table 3). Means are graphed in Figures 5 and 6, and individual data in Figure 7. The $d^{\prime}$ values increased as a function of TTP difference. By $t$ test, $d^{\prime}$ was significantly above chance for all TTP conditions $(p<.001)$. Values of $d^{\prime}$ did not vary as a function of radius. Mean $d^{\prime}$ was $0.72,0.76$, and 0.97 for radii of 40, 80, and $160 \mathrm{~m}$, respectively. Analysis of the bias showed no effect of TTP. Mean bias was -0.53 , $-0.53,-0.54$, and -0.5 for TTP differences of 0.25 , $0.5,0.75$, and $1 \mathrm{sec}$. However, bias decreased with ra- 
Table 2

Significant Within-Subjects Effects in Experiments 1, 2, 3, and 4

\begin{tabular}{crccc}
\hline Effect & $F$ & $d f$ & $p$ & $n /(n+\operatorname{missing})$ \\
\hline Time-to-passage & & & & \\
Experiment 1 & 6.16 & 3,18 & .0046 & $30 / 36$ \\
Experiment 2 & 18.24 & 3,20 & .0001 & $32 / 36$ \\
Experiment 3 & 10.91 & 3,23 & .0001 & $35 / 36$ \\
Experiment 4 & 3.14 & 3,15 & .0568 & $26 / 32$ \\
\hline
\end{tabular}

Note-The dependent measure was $d^{\prime}$. Because of ceiling effects, the number of observations $(n)$ was smaller than the expected total $(n+$ missing).

dius. For all radii, there was a significant bias to judge the target on the inside as passing by first $(p<.001)$.

\section{Discussion}

The results show that observers were able to make better than chance TTP judgments for targets approaching on a circular trajectory. Sensitivity to the information provided by global flow was reasonable. The overall performance was comparable with the performance in Kaiser and Mowafy's (1993) Experiment 1, in which PCR ranged from .55 with a 0.25 -sec temporal separation to .8 with a $1-\mathrm{sec}$ separation of leading and trailing targets. Because information in our displays was reduced to global flow, performance is expected to be lower than with displays that provide both global and local information. However, a comparison of our $d^{\prime}$ values with a comparable arrangement of two targets that provided both local and global expansion information (DeLucia \& Novak, 1997, Figure 2, collapsed across conflicting and corresponding pictorial cues) shows that sensitivity is in a similar range (overall $d^{\prime}$ of slightly less than 1 ). The striking result of the first experiment is that observers are sensitive to TTP information in displays that simulate circular approach. Tau-based theories predict that observers should not be able to extract such information at all.

The position of the leading target with respect to the observer's circular trajectory had a strong impact on correctness scores. Leading targets placed on the inside of the trajectory led to better performance, which indicates that the observers tended to select the inside target more often than the outside target. A separate bias analysis confirmed this conclusion. The inside bias decreased for larger radii. Also, targets placed far from the observer's trajectory were judged to pass by first more often than targets placed close to the trajectory. This result replicates the main finding of Kerzel et al. (1999), who demonstrated that targets offset by a large distance from the direction of motion appear closer to the observer than targets offset by a small distance. The optical velocity of far targets is higher, and presumably, observers use optical velocity as a cue to depth.

The above-chance performance and the inside bias shows that global tau was not used as an estimate of TTP. If the observers had relied on global tau, they should have consistently selected the target on the outside of the observer's trajectory, which always had smaller tau val- ues. This was clearly not the case. Quite to the contrary, the observers tended to select targets on the inside, which global tau specified to have longer arrival times. It can be concluded that observables other than tau must have guided the observers' judgments.

Although previous research (Kerzel et al., 1999) suggests that TTP judgments are largely based on the optical velocity of extensionless targets, the present results indicate that this is not the only cue to depth in environments void of size information. Targets placed outside of the observer's trajectory had consistently larger optical velocities. Nonetheless, the observers showed a strong tendency to select the target on the inside to pass by first. The most important difference between optical velocity profiles in linear approach scenarios (Kaiser \& Mowafy, 1993; Kerzel et al., 1999) and circular approaches is the ratio of optical acceleration and optical velocity, $A^{\prime \prime} / A^{\prime}$ (see Figure 3). For targets approaching on a linear trajectory, the relative optical acceleration is proportional to TTP, whereas this is not the case for nonlinear approaches. For observers moving on a circular trajectory, targets on the inside have smaller optical velocities and relatively large optical acceleration rates. On the other hand, targets on the outside have high optical velocities but relatively small optical acceleration rates. Thus, the tendency to judge the target on the inside of the trajectory as being closer indicates that observers relate high relative optical acceleration to nearness. If the observers had relied exclusively on optical velocity, as they do in linear cases, the opposite bias would have been expected. The results disprove this hypothesis.

Finally, if the observers had relied on absolute optical acceleration to judge TTP, we would expect no bias, because objects varying in the relative offsets had approximately equal acceleration rates. Thus, we conclude that usage of relative optical acceleration may explain the observed inside bias. Therefore, relative optical acceleration may be a cue to depth in circular approaches in addition to first order motion, which is also used in linear cases. Table 4 demonstrates that no single variable fully accounts for the inside bias observed in this experiment. If the observers had relied exclusively on relative acceleration rate, the inside target should have been selected in $95 \%$ of the cases (averaged across radii). However, the observers did so in $73 \%$ of all trials. Optical velocity predicted 28\% inside choices, global tau 0\% (averaged across radii). Global tau may therefore be considered the

Table 3

Significant Within-Subjects Effects in Experiments 1, 2, 3, and 4

\begin{tabular}{lrllc}
\hline \multicolumn{1}{c}{ Effect } & \multicolumn{1}{c}{$F$} & $d f$ & $p$ & $n /(n+$ missing $)$ \\
\hline Radius & & & & \\
Experiment 1 & 8.87 & 2,13 & .0037 & $24 / 27$ \\
Experiment 2 & 89.10 & 2,16 & .0001 & $27 / 27$ \\
Experiment 3 & 4.03 & 2,16 & .0382 & $27 / 27$ \\
Experiment 4 & 14.18 & 2,9 & .0017 & $19 / 24$ \\
\hline
\end{tabular}

Note-The dependent measure was bias. Because of ceiling effects, the number of observations $(n)$ was smaller than the expected total $(n+$ missing). 


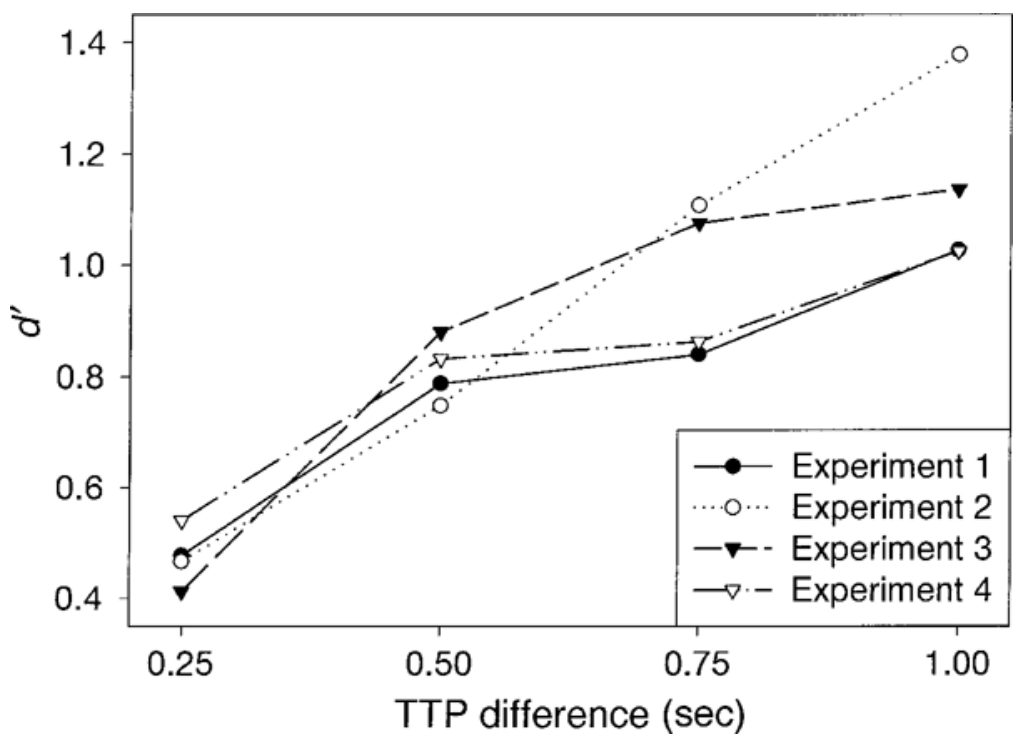

Figure 5. Mean $d^{\prime}$ values as a function of time-to-passage (TTP) difference and experiment.

poorest predictor of subjects' performance. Because neither optical velocity nor relative optical acceleration predicts the actual selection accurately, one may suggest that observers combine the two cues to judge relative arrival. This is evident in the influence of both distance and relative position with respect to the circular path on PCR.

However, it is important to note that only relative acceleration may explain the decrease of the inside bias with an increase in the radius of the trajectory. Inspec- tion of Figure 3 shows that the difference in relative acceleration rate between inside and outside targets decreases with an increase in radius. Therefore, if an observer exclusively relied on relative optical acceleration, the number of inside choices would be expected to diminish at larger radii (see Table 4). This is what was observed in the present experiment.

It remains to be explained why the observers may have used relative optical acceleration in the present experiment but failed to do so in the linear approach scenarios.

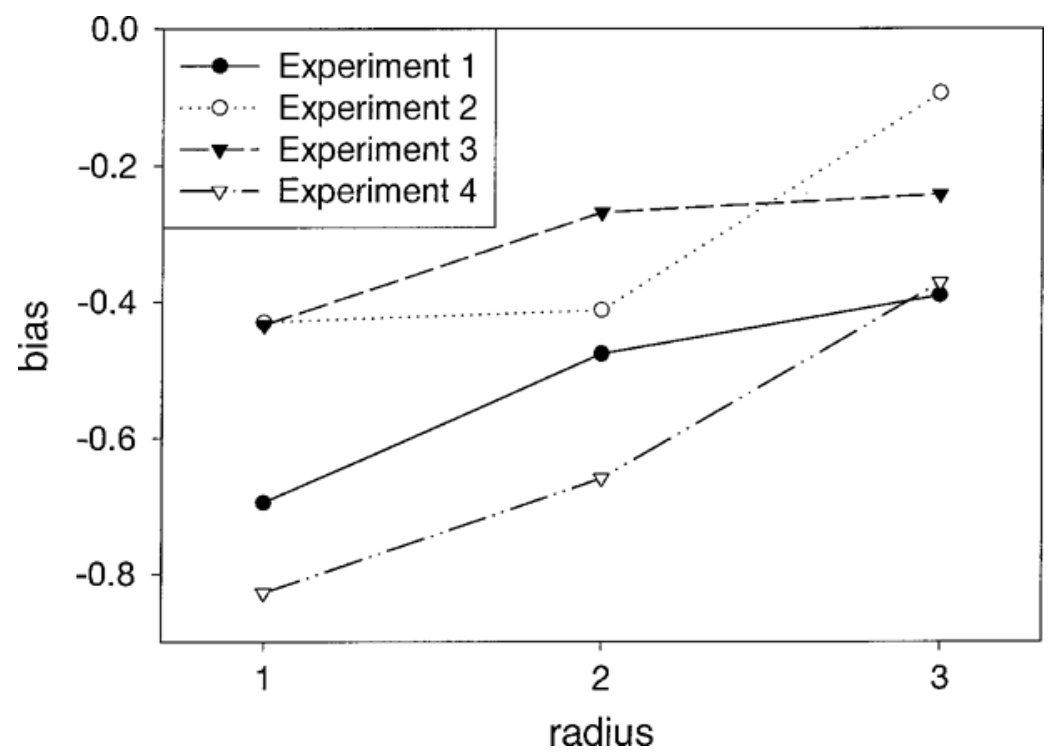

Figure 6. Mean bias values as a function of radius and experiment. The actual radii varied in the three experiments. Radii of 40, 80, and $160 \mathrm{~m}$ were used in Experiments 1 and 4, and radii of 120, 160, and $320 \mathrm{~m}$ were used in Experiments 2 and 3. Radii are depicted in ascending order-that is, radius 1 corresponds to the smallest radius. 


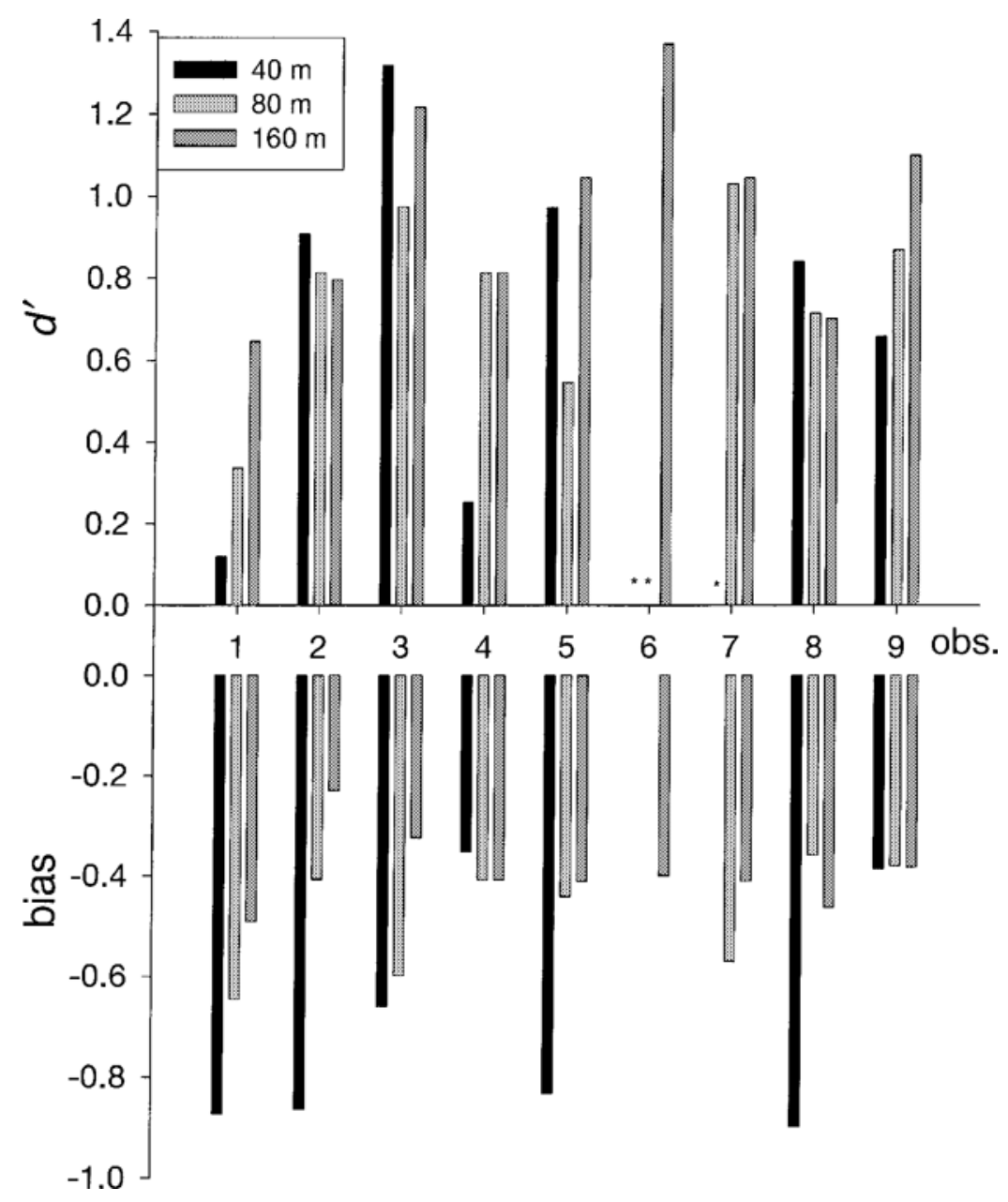

Figure 7. Individual data from Experiment 1. $d^{\prime}$ and bias values are shown for each observer. An asterisk indicates missing data owing to ceiling effects (hit rates of one).

For linear approaches, relative acceleration rate is a valid cue to depth, as Figure 1 demonstrates. Irrespective of target offset, relative optical acceleration is inversely proportional to TTP. Thus, if the observers had relied on this variable, no bias to select fast moving objects should have been observed by Kerzel et al. (1999). A possible solution to this problem is given in the absolute numbers. Comparison of Figures 2 and 3 shows that the relative

Table 4

Percentage of Trials in which Participants Selected the Target on the Inside of the Observer's Trajectory (Actual Inside Choices), With the Percentage of Trials in Which the Inside Target Had the Highest Optical Velocity, the Highest Relative Optical Acceleration, or the Smallest Global Tau Value

\begin{tabular}{|c|c|c|c|c|c|c|}
\hline \multirow{2}{*}{$\begin{array}{l}\text { Radius } \\
(\mathrm{m})\end{array}$} & \multirow{2}{*}{$\begin{array}{c}\text { Optical } \\
\text { Velocity } \\
(\%)\end{array}$} & \multirow{2}{*}{$\begin{array}{c}\text { Relative } \\
\text { Optical } \\
\text { Acceleration } \\
(\%) \\
\end{array}$} & \multirow{2}{*}{$\begin{array}{c}\text { Global } \\
\text { Tau } \\
(\%)\end{array}$} & \multicolumn{3}{|c|}{$\begin{array}{l}\text { Actual Inside Choices } \\
(\%)\end{array}$} \\
\hline & & & & Experiment 1 & Experiment 2 & Experiment 4 \\
\hline 40 & 16 & 100 & 0 & 80 & & 86 \\
\hline 80 & 28 & 100 & 0 & 72 & & 78 \\
\hline 120 & 38 & 91 & 0 & & 69 & \\
\hline 160 & 41 & 84 & 0 & 67 & 67 & 69 \\
\hline 320 & 44 & 57 & 19 & & 54 & \\
\hline
\end{tabular}

Note-Unsigned values were used in the comparison. The angle between tangential heading and target, its rate of change, and its acceleration were averaged over the last $250 \mathrm{msec}$ of the presentation. These values were used to calculate relative acceleration and global tau. 
optical acceleration for temporal differences of some seconds in the linear case is of a similar magnitude as the difference between inside and outside targets. This difference decreases with radius but is present even with large radii $(320 \mathrm{~m})$. Therefore, differences in relative optical acceleration between the inside and the outside targets were much more pronounced in the circular approach than in the linear approach. On the other hand, differences in optical velocity were somewhat larger in the linear case. Thus, the relative salience of the different cues may vary for linear and circular approaches. Differences in optical velocity were more pronounced in the linear case, whereas differences in relative optical acceleration were more pronounced in the circular case. Thus, it appears that observers consider relative optical acceleration when it is made more salient.

\section{EXPERIMENT 2 Large Circular Trajectories}

To investigate whether the inside bias vanishes for very large radii, we replicated Experiment 1 but added a trajectory with a $320-\mathrm{m}$ radius. With increasing radius, $r$, the angular velocity, $w$, decreases when the tangential velocity, $v$, remains constant, since $v=r * w$. Therefore, the nonlinear component introduced in the approach is reduced - that is, a smaller portion of the circle's phase is passed (see Figure 2B). Thus, with large radii, the linear case is approached, so that the difference in relative acceleration is further diminished. Therefore, observers should behave more or less as in the linear case, in which they judge fast-moving targets as passing by first.

\section{Method}

Subjects. Nine undergraduate students at the University of Connecticut participated in partial fulfillment of a course requirement. All the subjects had normal or corrected-to-normal vision.

Stimuli and Design. The same apparatus, design, and stimuli were used as those in Experiment 1, except that the radii were changed to 120,160 , and $320 \mathrm{~m}$.

\section{Results}

Proportion correct. Mean PCRs are graphed in Figure 8. A four-way within-subjects ANOVA $($ TTP $\times$ radius $\times$ relative position of leading target $X$ distance of leading target; see Table 1) showed that performance increased with TTP from .62 at the smallest to .83 at the largest temporal separation. Performance was above chance for all levels of TTP $(p<.005)$. PCR increased with radius from .69 at a radius of $120 \mathrm{~m}$ to .83 at $320 \mathrm{~m}$. Performance was superior when the leading target was inside the observer's trajectory (.85 vs. .59). More correct judgments were made when the leading target was close to the observers' trajectory (.81 vs. .62). The interaction between radius and position of the leading target was significant, indicating that the difference in performance between inside and outside placement of the leading target decreased from .38 at the smallest radius to .09 at the largest radius. By $t$ test, the advantage of the inside condition was significant even with the largest radius $(p<.0296)$. Also, the interaction between TTP differ-

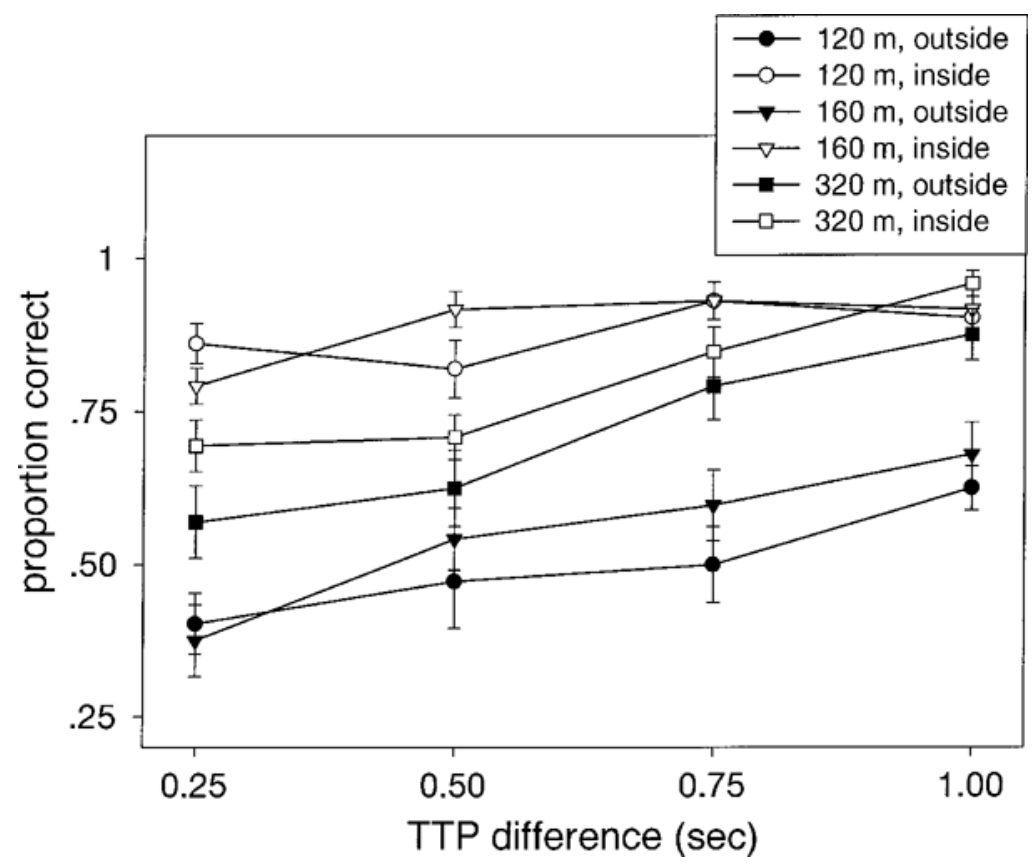

Figure 8. Mean proportion of correct responses and standard error (between subjects) as a function of relative position of the leading target, radius, and difference in time to passage (TTP) in Experiment 2. 
ence and position of the leading target reached significance, showing that the difference between inside and outside trials decreased with temporal separation from .33 at $0.25 \mathrm{sec}$ to .20 at $1 \mathrm{sec}$. Similarly, the advantage of targets placed close to the observer's trajectory decreased from .29 at $0.25 \mathrm{sec}$ separation to .12 at $1 \mathrm{sec}$. Furthermore, the position of the leading target interacted with the distance of the target. When the leading target was outside, the difference in performance between close and far placements was more pronounced (.47 vs. .7) than when the leading target was inside (.78 vs. .92). Overall, there was a significant bias to judge the inside target more often than the outside target to pass by first (63\% of all trials, different from $50 \%, p<.0001)$. Furthermore, targets far from the observer's trajectory were judged to pass by more often than targets close to the trajectory ( $59 \%$ of all trials, different from $50 \%, p<.0001)$.

$\boldsymbol{d}^{\prime}$ and bias. Mean $d^{\prime}$ and bias values are shown in Figures 5 and 6. Individual data are graphed in Figure 9. Across all conditions, mean $d^{\prime}$ was $0.94(S E=0.09)$, and mean bias was $-0.31(S E=0.03)$. One-way ANOVAs were conducted on $d^{\prime}$ (Table 2) and bias (Table 3) values, with TTP and radius as factors. Values of $d^{\prime}$ increased as a function of TTP difference. By $t$ test, $d^{\prime}$ was significantly above zero for all TTP conditions $(p<.001)$. The value of $d^{\prime}$ did not vary as a function of radius. Mean $d^{\prime}$ was $0.86,1$, and 1.04 for radii of 120,160 , and $320 \mathrm{~m}$. An analysis of the bias data showed that the inside bias decreased with radius. For radii of 160 and $320 \mathrm{~m}$, there was a significant bias to judge the target on the inside as passing by first $(p<.001)$. At the largest radius, this bias was only marginally significant $(p<.051)$. Bias did not vary as a function of TTP. For TTPs of 0.25, 0.5, 0.75, and $1 \mathrm{sec}$, the mean bias was $-0.33,-0.29,-0.32$, and -0.28 , respectively.

\section{Discussion}

Judgments of relative TTP were more accurate on large trajectories and with large temporal separations between the targets. Although the inside bias decreased with radius, it was still (marginally) significant at a radius of $320 \mathrm{~m}$. Therefore, even when the relative optical

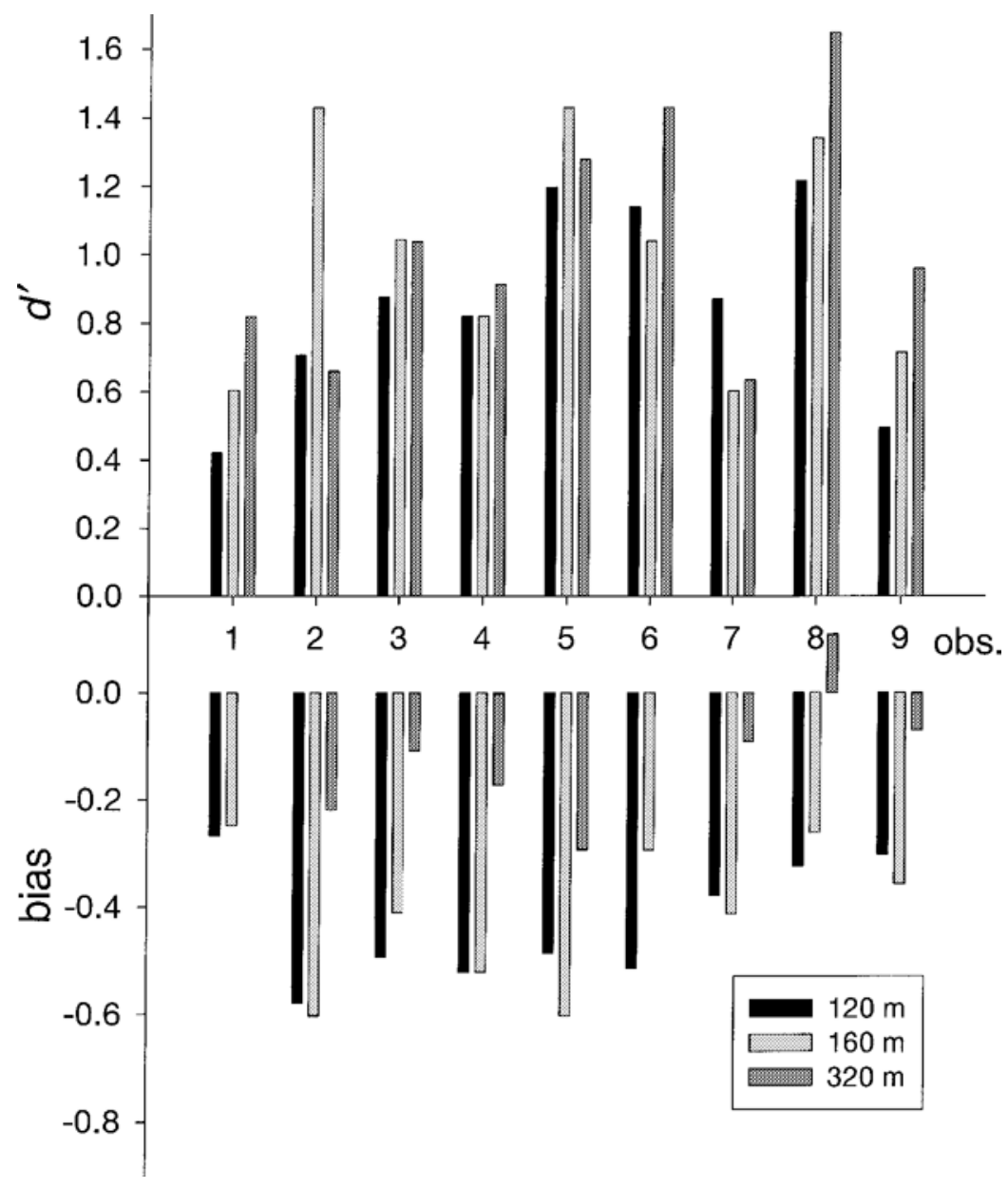

Figure 9. Individual data from Experiment 2. $d^{\prime}$ and bias values are shown for each observer. 
acceleration rates of inside and outside targets were almost identical (see Figure 3), the observers selected the target on the inside. However, the effect was small and appears less reliable than the inside bias observed with small radii. Nonetheless, the results show that even very large trajectories are different from the linear case and that subtle differences in relative optical acceleration suffice to induce a bias.

\section{EXPERIMENT 3 Relative Motion}

This experiment was conducted in order to rule out the possibility that the inside bias was attributable to a general tendency to select the target that was closer to the center of rotation. In Experiments 1-2, the two targets were always placed on different sides of the trajectory so that the inside target was always closer to the center of rotation. To disentangle distance from the center of rotation and position with respect to the trajectory, we placed the targets on the same side of the observer's trajectory. In this situation, the optical velocity profiles of leading and trailing targets are more similar than with the opposite placement. In particular, the relative acceleration rate is comparable for the two targets. However, the optical velocity of far targets remains higher, so that the observers are expected to select far targets as passing by first. In contrast, if the observers always selected the target closer to the center of rotation, they should select the target far from the observer's trajectory if both targets are placed on the inside and the target close to the trajectory if the two targets are placed on the outside.

\section{Method}

Subjects. Nine undergraduate students at the University of Connecticut participated in partial fulfillment of a course requirement. All the subjects had normal or corrected-to-normal vision.

Stimuli. The same apparatus and stimuli were used as those in Experiment 1.

Design. The first factor was the radius of the observer's trajectory. It was varied randomly between 120,160 , and $320 \mathrm{~m}$. The second factor was the position of the leading target. The leading target traveled either on the inside of the observer's trajectory or on the outside. The second factor was the targets' location with respect to the observer's trajectory. In each trial, target offsets of 25.4 and $50.8 \mathrm{~cm}$ from the track vector were represented - that is, one target was always offset by $25.4 \mathrm{~cm}$, and the other by $50.8 \mathrm{~cm}$. The targets appeared either on the same side of the track vector or on opposite sides of the track vector. The third factor was TTP difference. TTP differences of $0.25,0.5,0.75$, and $1 \mathrm{sec}$ were used.

Procedure. The same procedure as that in Experiment 1 was used.

\section{Results}

Proportion correct. Mean PCRs are graphed in Figure 10. Separate four-way ANOVAs $($ TTP $\times$ radius $\times$ relative position of leading target $\times$ leading target distance) were conducted for each target placement (see Table 1). When the targets were placed on different sides of the observer's trajectory, accuracy increased with TTP difference from .57 to .71 , and judgments were more accurate when the leading target was placed on the inside (.77 vs. .53). Responses were more accurate when the target was far from the trajectory (.82 vs. .47). Distance from the trajectory and position of the leading target interacted, showing that when the leading target was on the outside, the advantage of far targets was larger (.77 vs. $.27)$ than with inside placement (.88 vs. .67). Also, ra-

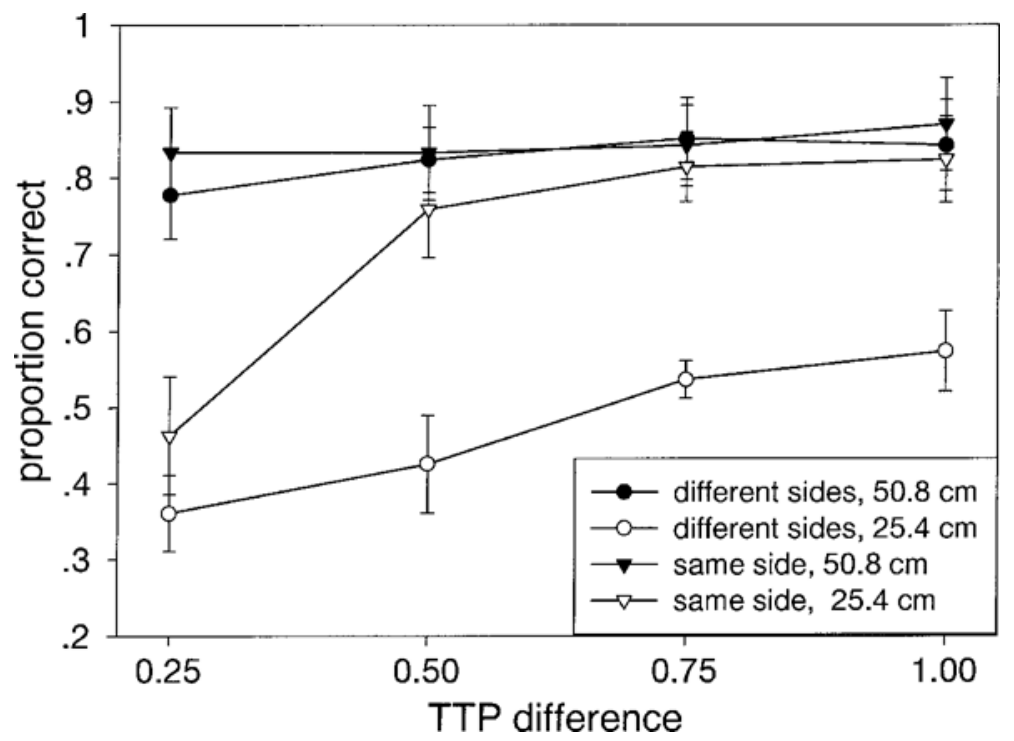

Figure 10. Mean proportion of correct responses and standard error (between subjects) as a function of distance of the leading target from the observer's trajectory, placement of the two target dots and time to passage (TTP) in Experiment 3. 


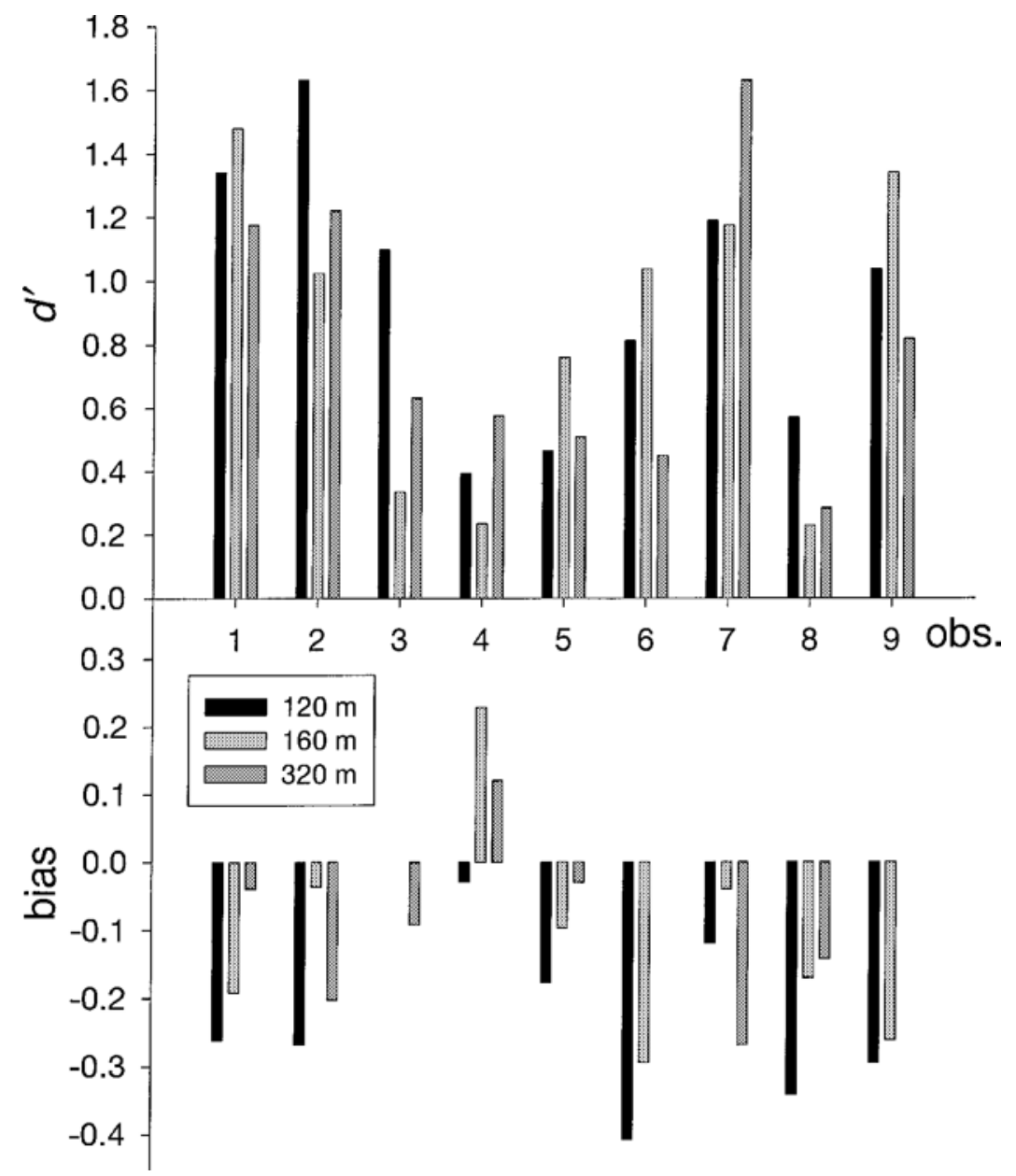

Figure 11. Individual data from Experiment 3. $d^{\prime}$ and bias values are shown for each observer.

dius and position of the leading target interacted, indicating that the advantage of trials with leading targets placed on the inside decreased from .35 at the smallest radius to .13 at the largest radius. In the same-side condition, there was a significant effect of TTP. Accuracy increased from .64 with a 0.25 -sec temporal separation to .85 with a 1 -sec separation. The subjects' answers were more accurate when the leading target was far from the trajectory (.84 vs. .72). Furthermore, distance from the trajectory interacted with TTP, indicating that the difference between leading targets placed close and far from the trajectory decreased with TTP from .37 with a 0.25 -sec to .05 with a 1 -sec temporal separation.

$\boldsymbol{d}^{\prime}$ and bias. Mean $d^{\prime}$ and bias values are shown in Figures 5 and 6. Individual data are graphed in Figure 11. Across all conditions, mean $d^{\prime}$ was $0.85(S E=0.13)$, and mean bias was $-0.12(S E=0.03)$. One-way ANOVAs were conducted to evaluate the effects of TTP and radius (see Tables 2 and 3). Values of $d^{\prime}$ increased as a function of TTP difference. By $t$ test, $d^{\prime}$ was significantly above zero for all TTP conditions $(p<.01)$. Values of $d^{\prime}$ did not vary as a function of radius. Mean $d^{\prime}$ was $0.95,0.85$, and 0.81 for radii of 120,160 , and $320 \mathrm{~m}$. The bias to select the target close to the center of rotation more often than the far decreased with radius. The inside bias was significant only for the smallest radius $(p<.0019)$. Separate analysis for the two target placement showed that the bias was present with opposite target placement $(M=$ $-0.25, S E=0.04, p<.0002)$, but not with same-side placement $(M=0.05, S E=0.04, p>.27)$. The bias did not vary with TTP. Mean bias was $-0.15,-0.17,-0.05$, and -0.09 , for TTPs of $0.25,0.5,0.75$, and $1 \mathrm{sec}$, respectively.

\section{Discussion}

In the present experiment, we fully replicated the findings from Experiments 1and 2. The subjects showed a strong tendency to select the target on the inside as passing by first. This bias decreased with the size of the radius. In addition, leading targets far from the trajectory were selected more often than leading targets that were close. In the condition in which the two targets were 
placed on identical sides of the trajectory, there was an advantage for leading targets far from the trajectory, irrespective of whether they were placed inside or outside of the trajectory. Thus, it is safe to conclude that the inside bias does not result from a tendency to select targets that are close to the center of rotation. If this had been the case, the observers should have selected targets close to the trajectory when both targets appeared on the outside. However, they perceived the far target as passing by first for both inside and outside placements of the targets.

\section{EXPERIMENT 4 Feedback and Dot Replacement}

So far, we have established that observers tended to select the target that moved on the inside of a circular trajectory as passing by first when asked to judge relative passage time. Tresilian (1995) hypothesized that with relative judgment tasks, observers may be prone to the usage of response strategies. Because we provided feedback in Experiments 1-3, the results may not accurately reflect observers' intuitions about arrival time, but one or another form of response strategy. In previous studies using similar displays and the same task, no effects of feedback have been observed (Kaiser \& Mowafy, 1993; Kerzel et al., 1999). That is, irrespective of whether subjects were told about when they were wrong, the same level of performance and the same biases were observed. However, in order to make sure that these factors do not account for the inside bias, Experiment 1 was rerun in the absence of feedback. Furthermore, it may have been the case that asymmetries in the number of dots present on the inside or outside were present in our displays. Dots that went out of sight were not replaced, so that a density gradient in depth may have resulted. Depth and the resulting differences in vector magnitudes have been shown to affect heading perception (Dyre \& Andersen, 1997) and may, therefore, also play a role in TTP judgments. To control for this factor, dots that went out of sight were replaced in the present experiment. If either the provision of feedback or the asymmetrical attrition of dots had produced the inside bias observed in Experiments 1-3, these manipulations were expected to eliminate the effect.

\section{Method}

Subjects. Eight students at the Ludwig-Maximilians University of Munich participated for pay. All the subjects had normal or corrected-to-normal vision.

Stimuli, Design, and Procedure. The stimuli, design, and procedure were the same as those in Experiment 1, with the following differences. The stimuli were generated by a P166 computer and a Matrox Mystique graphics adapter. Viewing distance was $50 \mathrm{~cm}$. Dots that went out of sight were repositioned at the far end of the dot volume at the same grid position (but with different jitter). The slightly asymmetric distribution of dots in Experiments 1-3 was avoided by assigning azimuth values from the same range to inside and outside dots. Dots with an azimuth smaller than $c=5.33 \mathrm{~m} / r$, with $r$ being the radius of the observer's path, were clipped. The azimuth values of dots in the cloud varied between $c$ and $c+11.4 \mathrm{~s} *$ $w$, with $w$ being the angular velocity. Thus, slightly less than half of the cloud's angular extend was traversed during the display duration $(5 \mathrm{sec})$. Vertical and horizontal deviations from the observer's path were $5 \mathrm{~m}$ horizontally and vertically. Thus, it was ensured that the observer traveled a radial distance that was a constant proportion of the cloud's radial extent and that dots were evenly distributed on the inside and outside. No feedback was given.

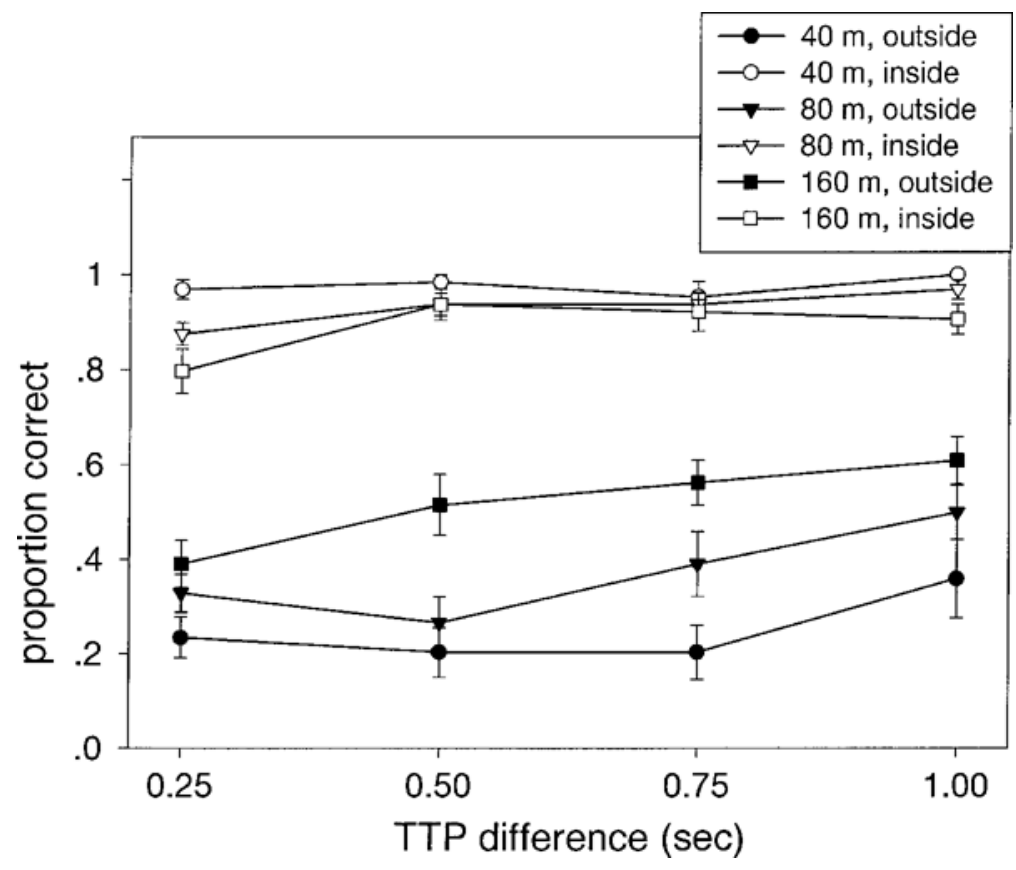

Figure 12. Mean proportion of correct responses and standard error (between subjects) as a function of relative position of the leading target, radius, and difference in time to passage (TTP) in Experiment 4. 


\section{Results}

Proportions correct. Mean PCRs are graphed in Figure 12. A four-way within-subjects ANOVA $($ TTP $\times$ radius $X$ relative position of leading target $X$ distance of leading target) was conducted (see Table 1). PCR increased with TTP difference from .60 at a 0.25 -sec temporal separation to .72 at a $1-\mathrm{sec}$ separation. By $t$ test, proportions correct were well above chance for all TTP differences $(p s<.0001)$. Accuracy increased with radius from .61 at $40 \mathrm{~m}$ to .71 at $160 \mathrm{~m}$. Judgments were more accurate when the leading target was on the inside than when it was on the outside of the observer's trajectory (.93 vs. .38). Accuracy was lower when the leading target was close to the observer's trajectory (.56 vs. .75). The advantage of inside targets was larger with close placement (.92 vs .2) than with far placement of the leading target (.94 vs. .57). The interaction of radius and the relative position of the leading target was significant, indicating that the advantage of trials with the leading targets on the inside decreased from .73 at $40 \mathrm{~m}$ to .37 at
$160 \mathrm{~m}$. Also, the difference between inside and outside targets decreased with TTP from .56 with a 0.25 -sec temporal separation to .47 with a $1-$ sec separation. The difference between far and close target placement increased with radius (.68 vs. .54 at $40 \mathrm{~m}, .83$ vs. .57 at $160 \mathrm{~m})$. Overall, there was a strong bias to judge the inside target as passing by first (77\% of all trials, different from $50 \%, p<.0001$ ). Furthermore, the target far from the observer's trajectory was judged to pass by first more often than the close target $(60 \%$ of all trials, different from $50 \%, p<.0001)$.

$\boldsymbol{d}^{\prime}$ and bias. Mean $d^{\prime}$ and bias values are shown in Figures 5 and 6. Individual data are graphed in Figure 13. Across all conditions, mean $d^{\prime}$ was $0.76(S E=0.1)$, and mean bias was $-0.65(S E=0.05)$. One-way ANOVAs on $d^{\prime}$ (Table 2) and bias (Table 3), with TTP and radius as factors, were conducted. TTP had a marginally significant effect on $d^{\prime}$. The values of $d^{\prime}$ tended to increase as a function of TTP difference. By $t$ test, $d^{\prime}$ was significantly above zero for all TTP conditions $(p s<.001)$. The

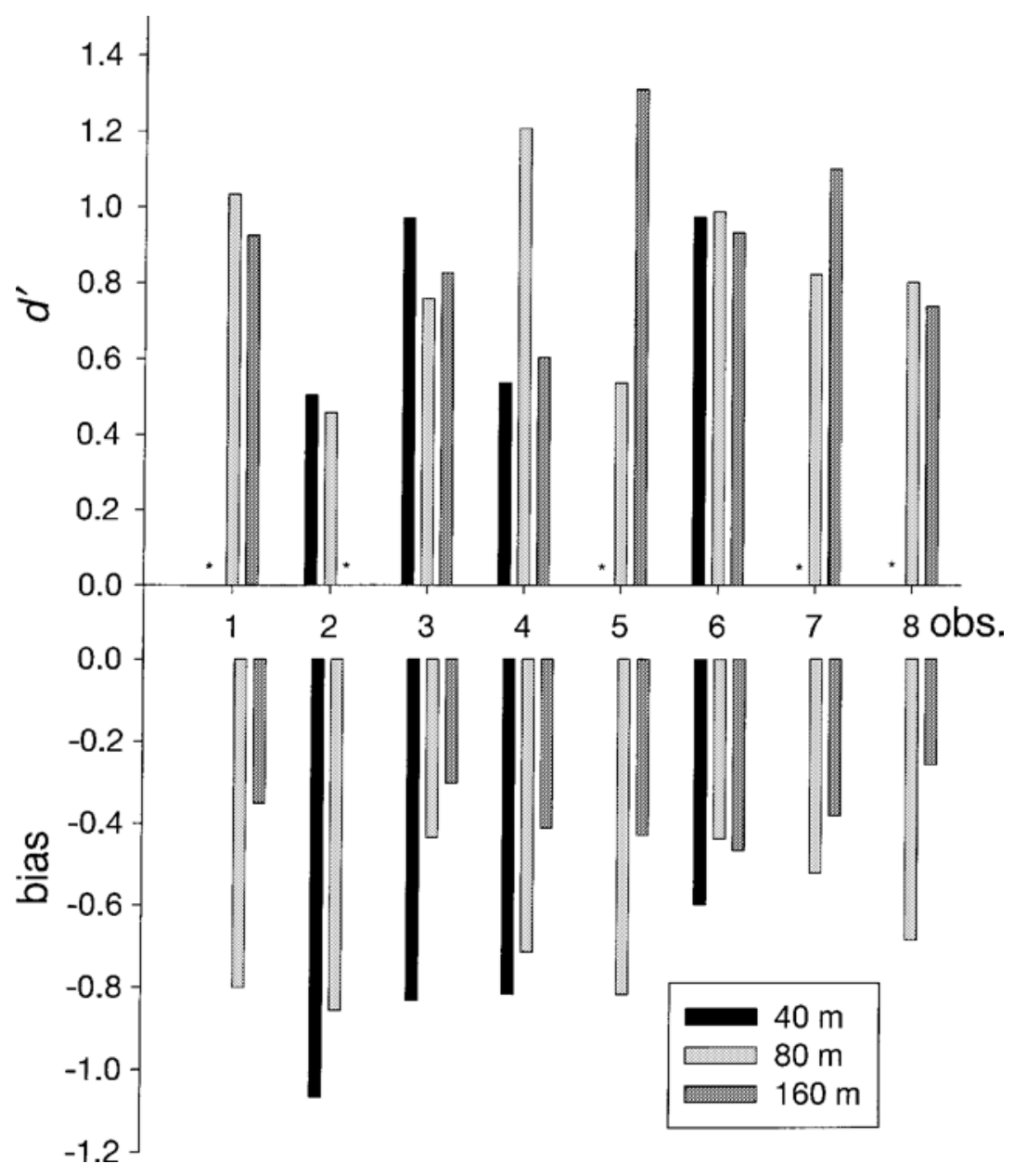

Figure 13. Individual data from Experiment 4. $d^{\prime}$ and bias values are shown for each observer. An asterisk indicates missing data owing to ceiling effects (hit rates of one). 
values of $d^{\prime}$ did not vary as a function of radius. Mean $d^{\prime}$ was $0.75,0.82$, and 0.92 for radii of 120,160 , and $320 \mathrm{~m}$. Analysis of the bias data showed that the inside bias decreased with radius but was significant for all radii ( $p$ s $<.005$ ). Bias did not vary as a function of TTP. For TTPs of $0.25,0.5,0.75$, and $1 \mathrm{sec}$, the mean bias was -0.61 , $-0.64,-0.66$, and -0.63 , respectively.

\section{Discussion}

The present experiment replicated the results of Experiment 1 . Therefore, neither the provision of feedback nor the potentially asymmetric attrition of dots may account for the inside bias. To the contrary, the inside bias was numerically larger in the absence of feedback $(-0.65$ in Experiment 4 vs. -0.53 in Experiment 1). These results are consistent with Kerzel et al. (1999), who reported no effect of feedback and showed that TTP judgments do not depend on the surrounding dots. In fact, their Experiment 4 showed that even the complete absence of a cloud did not alter TTP judgments.

\section{GENERAL DISCUSSION}

In the present study, we examined relative TTP judgments on circular trajectories. In contrast to linear trajectories, global tau does not provide reliable estimates of TTP for nonlinear approach. Therefore, it was surprising, from the point of view of current theories of interceptive timing, that observers were able to make above-chance judgments of relative arrival on both small and large trajectories. In research on linear approaches in an extensionless flow field (Kerzel et al., 1999), it was found that observers use optical velocity as a cue to depth. When global tau information and optical velocity were brought into conflict by varying the lateral offset of the targets, observers chose targets with a larger offset as passing by first, presumably because of their higher optical velocity. Global tau, on the other hand, is independent of target offset. In the present study, we replicated these findings: Leading targets that were close to the trajectory were less likely to be selected as passing by first. Similar to linear approach scenarios, optical velocity increases with distance from the trajectory, which may account for this bias.

Furthermore, observers also selected leading targets that were placed on the inside more often than leading targets on the outside. The bias decreased with radius but was still present even with large trajectories of $320 \mathrm{~m}$. This is surprising, given that inside targets have a lower optical velocity (and also larger global tau values). We suggest that observers' reliance on a cue of higher order, relative optical acceleration, explains this bias. Relative optical acceleration is a reliable predictor of TTP in linear scenarios, but not in circular ones. In circular approaches, targets placed on the inside have a higher relative optical acceleration rate irrespective of their TTP. Thus, reliance on this cue leads to erroneous judgments in circular approaches. The inside bias decreased as the size of the radius was increased. This result is accounted for by the fact that the ratio of relative optical acceleration rates for inside and outside targets approaches one with increasing radius. Relative optical acceleration is the simplest additional cue that, together with a sensitivity to optical velocity, can explain most of the variance in TTP judgments.

The inside bias found in our study is compatible with results obtained by Berthelon and Mestre (1993), who investigated a very specific flow field relating to driving. They presented computer-generated scenes from the perspective of a driver on a stretch of road that curved to the left. Observers had to judge whether a car approaching on a crossroad from the right would cross before or after the driver reached the intersection. Most errors (late judgments) were made when the other car would reach the crossing earlier than the driver. This bias is equivalent to the inside bias in the present experiments. Objects on the inside of the track vector were judged to arrive earlier than they actually did. Berthelon and Mestre attributed this bias to the lateral slip of the environment toward the outside of the curve. Accuracy increased with the addition of a road sign near the intersection that could serve as a reference mark. Thus, also in more realistic animations, which contained changes in optical size, shading, and texture, observers appeared to perceive objects on the inside of their trajectory to be closer. However, our explanation of the inside bias deviates from that of Berthelon and Mestre. We do not attribute it to the restricted field of view, which discloses a larger part of the outside of the trajectory, but to specific characteristics of optical velocities. As was demonstrated above, optical velocities differ with respect to both first order motion and the ratio of first and second order motion for targets on the inside and outside.

The bias to perceive the target on the inside of a trajectory as nearer is also similar to a pattern of results obtained by Warren et al. (1991; see Kerzel \& Hecht, 1997, for a replication, and Kim \& Turvey, 1998, for a failure to obtain this effect). When asked to make judgments about their future path on a circular trajectory, observers perceived their heading direction to be toward the inside of the actual trajectory. This bias was found to be more pronounced with smaller radii. Warren et al. (1991) examined a large number of hypotheses that attempt to explain misperception of heading toward the inside of circular paths that lead through a 3-D cloud. They concluded that no clear explanation of the error pattern can be provided, suspecting that the bias was an artifact of the restricted view of the flow pattern presented on a small display screen. Future research will have to show whether the bias in heading estimation can also be attributed to characteristics of optical motion. For instance, it might be the case that the high acceleration rate of the flow field is interpreted as a cue for the direction of motion.

In sum, global tau has been ruled out as a candidate to explain arrival time judgments on nonlinear trajectories. Targets with large offsets from the trajectory were judged 
to pass by first, presumably because their optical velocity is higher. Thus, large offsets are treated similarly to approaches on linear trajectories. Circular approaches, on the other hand, bear witness of a more sophisticated strategy. In addition to the tendency to perceive objects with a high optical velocity to be closer, observers showed better than expected performance when inside and outside target placements were compared. Targets on the inside of an observer's trajectory have a low optical velocity, but a high ratio of optical acceleration and optical velocity (relative optical acceleration), as compared with targets on the outside. This asymmetry diminishes with radius. Consistent with the assumption that relative optical acceleration is another cue to depth, observers showed a bias to judge targets placed on the inside as closer. This bias diminished with the size of the motion's radius. Thus, the visual processes underlying TTP estimation vary as a function of the complexity of the stimuli. In simple linear approach scenarios, a simple strategy based on optical velocity is used. In the more complex case of circular approach, this strategy is augmented by predictive information gleaned from relative optical acceleration.

\section{REFERENCES}

Berthelon, C., \& Mestre D. (1993). Curvilinear approach to an intersection and visual detection of collision. Human Factors, 35, 521534.

Bootsma, R. J., \& OudeJans, R. R. (1993). Visual information about time-to-collision between two objects. Journal of Experimental Psychology: Human Perception \& Performance, 19, 1041-1052.

Bootsma, R. J., \& VAN Wieringen, P. C. W. (1990). Timing an attacking forehand in table tennis. Journal of Experimental Psychology: Human Perception \& Performance, 16, 21-29.

Cutting, J. E. (1986). Perception with an eye for motion. Cambridge, MA: MIT Press.

DeLucia, P. R. (1991). Pictorial and motion-based information for depth perception. Journal of Experimental Psychology: Human Perception \& Performance, 17, 738-748.

DeLucia, P. R., \& LidDell, G. W. (1998). Cognitive motion extrapolation and cognitive clocking in prediction motion tasks. Journal of Experimental Psychology: Human Perception \& Performance, 24, 901-914.

DeLucia, P. R, \& Novak, J. B. (1997). Judgments of relative time-tocontact of more than two approaching objects: Toward a method. Perception \& Psychophysics, 59, 913-928.

Delucia, P. R., \& Warren, R. (1994). Pictorial and motion-based information during active control of self-motion: Size-arrival effects on collision avoidance. Journal of Experimental Psychology: Human Perception \& Performance, 20, 783-798.

Dy RE, B. P., \& ANDERSEN, G. J. (1997). Image velocity magnitudes and perception of heading. Journal of Experimental Psychology: Human Perception \& Performance, 23, 546-565.

GiBson, J. J. (1966). The senses considered as perceptual system. Boston: Houghton Mifflin.

HEUER, H. (1993). Estimates of time to contact based on changing size and changing target vergence. Perception, 22, 549-563.

Hoy LE, F. (1957). The black cloud. London: Heineman.

Kaiser, M. K., \& Hecht, H. (1995). Time-to-passage judgments in nonconstant optical flow fields. Perception \& Psychophysics, 57, 817-825.

Kaiser, M. K., \& Mowafy, L. (1993). Optical specification of time-topassage: Observers' sensitivity to global tau. Journal of Experimental Psychology: Human Perception \& Performance, 19, 1028-1040.

Kerzel, D., \& Hecht, H. (1997). Heading backward: Perceived direction of movement in contracting and expanding optical flow fields. Psychonomic Bulletin \& Review, 4, 516-523.

Kerzel, D., Hecht, H., \& Kim, N.-G. (1999). Image velocity, not tau, explains arrival time judgments from global optical flow. Journal of Experimental Psychology: Human Perception \& Performance, 25, $1-16$.

KIM, N.-G., \& Turvey, M. T. (1998). Visually perceiving heading on circular and elliptical paths. Journal of Experimental Psychology: Human Perception \& Performance, 24, 1690-1704.

Law, D. J., Pellegrino, J. W., Mitchell, S. R., Fischer, S. C., McDonald, T. P., \& Hunt, E. B. (1993). Perceptual and cognitive factors governing performance in comparative arrival-time judgments. Journal of Experimental Psychology: Human Perception \& Performance, 19, 1183-1199.

LEE, D. N. (1976). A theory of visual control of braking based on information about time-to-collision. Perception, 5, 437-459.

LeE, D. N., Young, D. S., \& Rewt, D. (1992). How do somersaulters land on their feet? Journal of Experimental Psychology: Human Perception \& Performance, 18, 1195-1202.

Macmillan, N. A., \& Creelman, C. D. (1991). Detection theory: A user's guide. Cambridge: Cambridge University Press.

Schiff, W., \& Detwiler, M. L. (1979). Information used in judging impending collision. Perception, 8, 647-658.

SchifF, W., \& OldAK, R. (1990). Accuracy of judging time to arrival: Effects of modality, trajectory, and gender. Journal of Experimental Psychology: Human Perception \& Performance, 16, 303-316.

Simpson, W. A. (1988). Depth discrimination from optic flow. Perception, 17, 497-512.

Stone, L. S., \& Perrone, J. A. (1997). Human heading estimation during visually simulated curvilinear motion. Vision Research, 37, 573 590.

ToDD, J. T. (1981). Visual information about moving objects. Journal of Experimental Psychology: Human Perception \& Performance, 7, 795-810.

Tresilian, J. R. (1991). Empirical and theoretical issues in the perception of time to contact. Journal of Experimental Psychology: Human Perception \& Performance, 17, 865-876.

Tresilian, J. R. (1995). Perceptual and cognitive processes in time-tocontact estimation: Analysis of prediction-motion and relative judgment tasks. Perception \& Psychophysics, 57, 231-245.

WANN, J. P. (1996). Anticipating arrival: Is the tau margin a specious theory? Journal of Experimental Psychology: Human Perception \& Performance, 22, 1031-1048.

Warren, W. H., Mestre, D. R., Blackwell, A. W., \& Morris, M. W. (1991). Perception of circular heading from optical flow. Journal of Experimental Psychology: Human Perception \& Performance, 17, $28-43$.

Warren, W. H., Morris, M. W., \& Kalish, M. (1988). Perception of translational heading from optical flow. Journal of Experimental Psychology: Human Perception \& Performance, 14, 646-660.

(Manuscript received December 13, 1999; revision accepted for publication January 23, 2001.) 